\title{
SCHWARZ'S LEMMA AND THE SZEGÖ KERNEL FUNCTION
}

\author{
BY \\ P. R. GARABEDIAN( ${ }^{(1)}$
}

Introduction. This paper is concerned with extremal problems in the family of bounded analytic functions in a multiply-connected domain $D$, and it is concerned with extremal problems for the mean modulus $\Phi_{C}|f| d s$ of meromorphic functions $f$ in $D$ taken over the boundary $C$ of $D$. These two types of problems are shown to be closely related, and solutions are obtained simultaneously for both types by a method of contour integration. An altogether analogous method was exploited by Grunsky in his thesis $[8]\left({ }^{2}\right)$ for the investigation of schlicht functions in a multiply-connected domain. Thus it is interesting to remark that the fundamental distortion theorems of schlicht conformal mapping theory have been developed by Grunsky by a method which we are able to apply here to obtain the fundamental distortion theorems for bounded functions. It will appear, then, that the generalization of Schwarz's lemma to multiply-connected domains and the generalization of the Koebe distortion theorem can be carried out by a unified technique $\left({ }^{3}\right)$.

We shall find in addition to this that, while the recent papers of Bergman and Schiffer $[5,16]$ have developed a close relationship between the theory of schlicht canonical mappings and the Bergman kernel function [3], we are able to develop here a relationship between the theory of bounded functions and the Szegö kernel function [19]. Thus the Szegö kernel function does for the theory of distortion of bounded functions what the Bergman kernel function does for the theory of distortion of schlicht functions. We point out that both these kernel functions are actually differentials, and that in the Szegö case one is dealing with length and in the Bergman case one is dealing with area. Thus the mean modulus $\oint_{C}|f| d s$, or $\iint_{D}|f|^{2} d x d y$, should be thought of as a length, or area, and not as a mean modulus.

All these remarkable relationships are brought to light by using the simple boundary relations satisfied by the classical domain functions, such as

Presented to the Society, April 17, 1948; received by the editors May 19, 1948.

(1) Paper done with the assistance of contract N5 ori 76-16, NR 043-046 with the Office for Naval Research.

(2) Numbers in brackets refer to the bibliography at the end of the paper.

(8) The referee has called the author's attention to the work of F. Riesz, Über Potenzreihen mit vorgeschriebenen Anfangsgliedern, Acta Math. vol. 42 (1920) pp. 145-171, in which extremal problems for $\Phi|f| d s$, taken over the unit circle, are related by the same method of contour integration which we use here to the coefficient problem of bounded functions in the unit circle. Riesz's results, which he proves by special techniques applicable only in the unit circle, play relative to the present paper the same role that the classical area principle in simply-connected domains plays for Grunsky's thesis. 
Green's function, and their derivatives. We merely express quantities upon which we intend to obtain bounds by means of the Cauchy integral theorem, and use the boundary relations in analyzing the resulting contour integrals. However, it is not always easy to show the existence of the necessary domain functions for this process in the case of bounded functions. Thus we require in most cases the use of the variational technique of Grunsky [9] and Ahlfors [1], to whom the most far-reaching work on Schwarz's lemma is due. Following Ahlfors, we solve a variational problem for a simple subclass of the class of all bounded analytic functions, and utilize the properties of the extremal function of the subclass, which we find by the variational method, in order to use the method of contour integration. This technique follows the pattern of the calculus of variations, where one sets up a field of extremals satisfying Euler's differential equation and thus proves an inequality. The Ahlfors variational method yields precisely the material necessary for the proof of the inequality we are seeking.

We give, in the simplest case, an alternative proof of existence of the necessary domain functions which depends upon the Jacobi inversion problem for Abelian integrals. This proof has some importance in finding the extremal functions for our problems explicitly, but many of the more interesting problems in bounded functions, for example, the generalization of the Pick-Nevanlinna interpolation theory to multiply-connected domains, lead to such complicated generalizations of the Jacobi problem that we are not able to base a satisfactory theory upon this method of attack.

One of the most interesting theorems which we are able to prove as a consequence of our results concerning bounded functions is that the upper bound in Schwarz's lemma can be expressed in terms of the Szegö kernel function. Since, as Bergman has pointed out, the kernel functions of complete orthonormal systems of analytic functions are among the most easily computed domain functions in conformal mapping, we have here a result of practical importance. We obtain one of the most effective necessary and sufficient conditions known for the solution of the problem of Painleve concerning the existence of bounded, non-constant analytic functions in the exterior of a given compact set.

We have pointed out that the mean modulus $\oint_{C}|f| d s$ of meromorphic functions $f$ in $D$ is related to the length of the image of $D$ in conformal mapping, a fact which will be evident to those who are familiar with Szegö's paper [19]. We note that from this view point the variational method which Schiffer has used on extremal problems involving area [15] leads to a formal but highly instructive motivation of our work. Application of this method shows intuitively that the problem of minimum length analogous to Schiffer's problem of maximum area is related to extremal maps on Riemann surfaces bounded by non-concentric circles of unit radius. We note in this connection that the formulas derived in this paper for the Ahlfors variation of zeros of 
extremal bounded functions are parallel to the relations derived by Garabedian and Schiffer [7] for the Schiffer variational method.

We summarize the contents of the paper. In Chapters I and II known material is developed which is perhaps not standard technique for the modern student, but is essential to an understanding of the proofs we shall give further on. In Chapter III the method of contour integration is used to extend Schwarz's lemma, and the relation to the Jacobi problem is developed. In Chapter IV we determine the Szegö kernel function in terms of the more standard domain functions, and show the relations which exist between this function and Schwarz's lemma. Formulas are derived relating the Szegö and Bergman kernel functions. Chapter $\mathrm{V}$ is devoted to an application of these results to the problem of Painlevé. In Chapter VI we extend the PickNevanlinna theory of interpolation [20] to multiply-connected domains; for the implications of this result, we refer to the applications by Heins [11] of this theory for simply-connected domains. In the final Chapter VII, we treat a problem for meromorphic functions with bounded boundary values.

I. The Jacobi inversion problem. In this chapter we state a number of fundamental results concerning the Jacobi inversion problem in the theory of Abelian integrals. We shall have use for these results later in this paper. For a detailed discussion and for proofs we refer to Neumann [12].

Let $S$ be a closed Riemann surface of genus $p$, and denote by $a_{1}, \cdots, a_{p}$ and $b_{1}, \cdots, b_{p}$ a system of canonical cuts on $S$. Let $u_{1}, \cdots, u_{p}$ be the normalized set of integrals of the first kind on $S$ such that each $u_{\nu}$ has a period $\delta_{\nu \mu} \pi i$ over any closed path on $S$ crossing $a_{\mu}$ once and crossing no other canonical cut, where $\delta_{\nu \mu}=0$ for $\nu \neq \mu$ and $\delta_{\nu \nu}=1$. We denote the period of $u_{\nu}$ on a closed path crossing $b_{\mu}$, but not crossing any other canonical cut, by $b_{\mu \nu}$, $\nu, \mu=1, \cdots, p$. The functions $u_{1}, \cdots, u_{p}$ are single-valued on the surface $S_{a b}$ obtained from $S$ by deleting the canonical cuts $a_{1}, \cdots, a_{p}, b_{1}, \cdots, b_{p}$.

We define the Riemann theta-function

$$
\theta\left\{u_{1}, \cdots, u_{p}\right\}=\sum_{m_{1}, \ldots, m_{p=-\infty}}^{\infty} \exp \left(\sum_{i, j=1}^{p} b_{i j} m_{i} m_{j}+2 \sum_{i=1}^{p} u_{i} m_{i}\right)
$$

corresponding to the Riemann surface $S$. The series representing $\theta\left\{\cdots, u_{k}, \cdots\right\}$ converges for all values of the $u_{k}$, as can be shown by using the fact that the quadratic form

$$
\sum_{i, j=1}^{p} \operatorname{Re}\left\{b_{i j}\right\} m_{i} m_{j}
$$

is negative-definite.

We suppose that $S$ is represented as a covering surface of the $\zeta$-plane of $q$ sheets with suitable branch-points. On this surface $S$ we seek to determine $p$ points $\zeta_{1}, \cdots, \zeta_{p}$ satisfying the system of equations 


$$
\sum_{\mu=1}^{p} u_{\nu}\left(\zeta_{\mu}\right)=V_{\nu}, \quad \nu=1, \cdots, p .
$$

This is the inversion problem of Jacobi, in which it is desired to find the $\zeta_{\mu}$ on $S$ as functions of the $V_{\nu}$. It is most convenient to determine one branch of the functions $u_{v}$ on $S_{a b}$ and then to add suitable periods of these integrals to the $V_{\nu}$ so that no loss of generality is involved in thus restricting the $u_{v}$. That is to say, it is sufficient to solve the problem

$$
\sum_{\mu=1}^{p} u_{\nu}\left(\zeta_{\mu}\right)=V_{\nu}+r_{\nu} \pi i+\sum_{\mu=1}^{p} s_{\mu} b_{\mu \nu}, \quad \nu=1, \cdots, p,
$$

on $S_{a b}$ in order to solve the problem (3) on $S$, where the $r_{\nu}$ and $s_{\mu}$ are properly chosen integers.

It is found that a solution of the Jacobi problem always exists, and that either this solution is unique or else one $\zeta_{\mu}$ in the solution can be chosen arbitrarily.

One method of determining the complex values in the $\zeta$-plane of the points $\zeta_{\mu}$ on $S$ is the following. We let $A$ and $B$ be two complex numbers which are covered on $S$, respectively, by points $\alpha_{1}, \cdots, \alpha_{p}$ and by points $\beta_{1}, \cdots, \beta_{p}$. We suppose that the $\alpha_{\nu}$ can be connected to the $\beta_{\nu}$ by paths $\Omega_{\nu}$ on $S$ which do not cut the canonical slits $b_{1}, \cdots, b_{p}$. We pick any $p$ points $x_{1}, \cdots, x_{p}$ of $S_{a b}$ and set

$$
X_{\nu}=\sum_{\mu=1}^{p} u_{\nu}\left(x_{\mu}\right), \quad \nu=1, \cdots, p .
$$

Then the $\zeta_{\mu}$ determined from the system of equations (4) satisfy the condition

$$
\begin{aligned}
& \prod_{\mu=1}^{p} \frac{\left(\zeta_{\mu}-A\right)\left(x_{\mu}-B\right)}{\left(\zeta_{\mu}-B\right)\left(x_{\mu}-A\right)} \\
& \quad=\prod_{\mu=1}^{q} \frac{\theta\left\{\cdots, V_{k}-u_{k}\left(\alpha_{\mu}\right), \cdots\right\} \theta\left\{\cdots, X_{k}-u_{k}\left(\beta_{\mu}\right), \cdots\right\}}{\theta\left\{\cdots, V_{k}-u_{k}\left(\beta_{\mu}\right), \cdots\right\} \theta\left\{\cdots, X_{k}-u_{k}\left(\alpha_{\mu}\right), \cdots\right\}} .
\end{aligned}
$$

For $p$ different choices of the constants $A, B$ we obtain $p$ equations for the determination of the $\zeta_{\mu}$. Of course, these equations will not be independent in the case when the $\zeta_{\mu}$ are not uniquely determined.

We remark that it is necessary here to choose the arbitrary constants involved in the normal integrals of the first kind $u_{\nu}(\zeta)$ in a suitable fashion in order that the equations we have written be valid in this simple form. This choice is made by requiring that the Riemann constants $K_{1}, \cdots, K_{p}$ satisfy relations of the form

$$
K_{\nu}=\tilde{r}_{\nu} \pi i+\sum_{\mu=1}^{p} \tilde{s}_{\mu} b_{\mu \nu}, \quad \nu=1, \cdots, p
$$


where $\tilde{r}_{\nu}$ and $\tilde{s}_{\mu}$ are integers. The $K_{\nu}$ are defined by

$$
\begin{aligned}
K_{\nu}=\frac{u_{\nu}\left(\sigma_{\nu}\right)+u_{\nu}\left(\tau_{\nu}\right)}{2}+\sum_{\mu=1}^{p}\left\{\frac{b_{\mu \nu}}{2}+\frac{1}{\pi i} \oint_{b_{\mu}} \frac{u_{\nu}(\lambda)+u_{\nu}(\rho)}{2} d u_{\mu}\right\}, & \\
\nu & =1, \cdots, p,
\end{aligned}
$$

where $\lambda$ and $\rho$ lie on opposite sides of the cut $b_{\mu}$ on $S_{a b}, \mu=1, \cdots, p$, and $\sigma_{\nu}$ and $\lambda_{\nu}$ lie on opposite sides of the cut $b_{\nu}$ on $S_{a b}$ at the intersection of $b_{\nu}$ with $a_{\nu}, \nu=1, \cdots, p$. For $p \geqq 2$ the constants involved in the definition of the $u_{\nu}$ can be chosen so that the conditions ( 7$)$ are satisfied. The case $p=1$ leads, of course, to the far simpler problem of the inversion of elliptic integrals, which we shall not discuss.

We point out that it is more convenient to express our problem in the equivalent form

$$
\sum_{\mu=1}^{p} \int_{x_{\mu}}^{\zeta_{\mu}} d u_{\nu}=V_{\nu}-X_{\nu}, \quad \nu=1, \cdots, p,
$$

and in this way we avoid all topological discussion of the periods, since the path of integration can be chosen to have a homology type such that these cancel. Finally, if the determinant

$$
\left\|\frac{d u_{\nu}\left(\zeta_{\mu}\right)}{d \zeta_{\mu}}\right\|
$$

does not vanish, we can apply the implicit function theorem to investigate solutions of (3) for values of $V$, near the initial values.

II. The method of Lagrange multipliers. We shall prove in some detail a necessary condition for a real function of several real variables to have a minimum when conditions are imposed upon the manifold over which the real variables range. Our purpose is to express the well known results on Lagrange multipliers in a form convenient for applications in this paper.

Let $u(x)=u\left(x_{1}, \cdots, x_{n}\right), v_{1}(x)=v_{1}\left(x_{1}, \cdots, x_{n}\right), \cdots, v_{m}(x)=v_{m}\left(x_{1}, \cdots\right.$, $x_{n}$ ) be real functions of the real variables $x_{1}, \cdots, x_{n}$ which are continuous with all their derivatives of the first two orders in a neighborhood of the origin. Suppose that

$$
u(0)=v_{1}(0)=\cdots=v_{m}(0)=0
$$

and suppose that

$$
u(x) \geqq 0
$$

for small values of the $x_{i}$ satisfying the conditions

$$
\begin{array}{rlrl}
v_{j}(x) & =0, & j=1, \cdots, m, \\
x_{i} \geqq 0, & i=1, \cdots, n .
\end{array}
$$


That is, suppose that $u(x)$ has a relative minimum at the origin under the conditions (13) and (14). Then there exist real numbers $\lambda_{0}, \lambda_{1}, \cdots, \lambda_{m}$, not all zero, such that

$$
\lambda_{0} \frac{\partial u}{\partial x_{i}}+\sum_{j=1}^{m} \lambda_{j} \frac{\partial v_{j}}{\partial x_{i}} \leqq 0, \quad i=1, \cdots, n,
$$

where the partial derivatives are to be evaluated at the origin.

The proof is most easily carried out by a geometrical discussion of the location of the vectors

$$
U_{i}=\left[\frac{\partial u}{\partial x_{i}}, \frac{\partial v_{1}}{\partial x_{i}}, \cdots, \frac{\partial v_{m}}{\partial x_{i}}\right], \quad i=1, \cdots, n,
$$

in the $(m+1)$-dimensional Euclidean space $E_{m+1}$ of coordinates $\left[y_{0}, \cdots, y_{m}\right]$. We wish to prove that there is a vector $\lambda=\left[\lambda_{0}, \cdots, \lambda_{m}\right]$ in $E_{m+1}$ making an angle of not less than ninety degrees with each of the vectors $U_{i}$. It suffices to show that the $U_{i}$ all lie in a half-space of $E_{m+1}$ situated on one side of a hyperplane $E_{m}$ through the origin.

Suppose this is not the case. Then the linear combinations

$$
U=\sum_{i=1}^{n} x_{i} U_{i}
$$

of the $U_{i}$ with positive coefficients $x_{i}$ span the entire space $E_{m+1}$. In particular, we can find vectors of the form $U$ which describe the surface $S_{m}$ of a small $m$-sphere in $E_{m+1}$. For the corresponding values of the $x_{i}$, we find that the vector $\left[u(x), v_{1}(x), \cdots, v_{m}(x)\right]$ describes a surface $\Sigma_{m}$ close to $S_{m}$. The definition of $\Sigma_{m}$ is made precise by associating with each point of $S_{m}$ a vector $U$ whose coefficients $x_{i}$ are successively normalized to be as small as possible. That is, if the representation (17) of $U$ is not uniquely determined we minimize $x_{1}$. If this does not fix the representation, we minimize $x_{2}$ with $x_{1}$ already a minimum, and so on. We use these minimal $x_{i}$ in forming $\Sigma_{m}$. They are seen to depend continuously upon our point of $S_{m}$. For example, $x_{1}$ is the distance of the point $U$ of $S_{m}$ to the space spanned by the vectors $U_{2}, \cdots, U_{n}$, measured in the direction of $U_{1}$ and divided by the length of $U_{1}$. Such a distance is a continuous function of $U$. Note that we mean by the space spanned by $U_{2}, \cdots, U_{n}$ the space of combinations (17) with $x_{1}=0, x_{2}$ $\geqq 0, \cdots, x_{n} \geqq 0$. The continuity proof for the remaining $x_{i}$ can be carried out thus after continued projection of $S_{m}$ into the space of the $U_{j}$ with $j \geqq i$.

We find from the continuity of the $x_{i}$ that $\Sigma_{m}$ is a continuous image of $S_{m}$. $\Sigma_{m}$ is also close to $S_{m}$, since we have by Taylor's theorem with remainder

$$
u(x)=\sum_{i=1}^{n} x_{i} \frac{\partial u}{\partial x_{i}}+o(x)
$$




$$
v_{j}(x)=\sum_{i=1}^{n} x_{i} \frac{\partial v_{j}}{\partial x_{i}}+o(x), \quad j=1, \cdots, m,
$$

where the symbol $o(x)$ denotes terms of higher order than the first in the variables $x_{i}$. Hence, if the sphere $S_{m}$ is sufficiently small, the surface $\Sigma_{m}$ will enclose the origin, and there will exist a point $x=\left[x_{1}, \cdots, x_{n}\right]$ with nonnegative coordinates $x_{i}$ such that $\left[u(x), v_{1}(x), \cdots, v_{m}(x)\right]$ lies on the negative $y_{0}$-axis in $E_{m+1}$. The rigorous formulation of these intuitive statements can be made using the topological notions of homotopy and the degree of a mapping [2]. Our condition may be expressed in the form

$$
\begin{aligned}
u(x) & <0, \\
v_{j}(x) & =0, \\
x_{i} & \geqq 0,
\end{aligned}
$$

$$
\begin{gathered}
j=1, \cdots, m, \\
i=1, \cdots, n .
\end{gathered}
$$

This contradicts the hypotheses (12), (13), and (14). Hence the vector $\lambda \neq 0$ exists satisfying (15).

Note that if for some $x_{i}$ the condition (14) can be dropped, then in the corresponding relation (15) equality holds.

III. Schwarz's Lemma. Let $D$ be a finite domain of the z-plane bounded by $n$ analytic curves $C_{1}, C_{2}, \cdots, C_{n}$, and denote by $C$ the entire boundary, $\sum_{\nu=1}^{n} C_{\nu}$, of $D$. Let $\mathcal{F}_{z_{1}}$ be the class of functions $f(z)$ regular in $D$ and continuous in $D+C$, except at the point $z_{1} \in D$, where $f(z)$ is to have an expansion

$$
f(z)=\frac{1}{\left(z-z_{1}\right)^{2}}+\frac{a_{-1}}{\left(z-z_{1}\right)}+a_{0}+a_{1}\left(z-z_{1}\right)+\cdots
$$

Let $\Omega_{z_{1}}$ be the class of analytic functions $F(z)$ in $D$ satisfying $|F(z)| \leqq 1$. there and with expansions

$$
F(z)=\alpha_{F}\left(z-z_{1}\right)+b_{1}\left(z-z_{1}\right)^{2}+\cdots, \alpha_{F}>0,
$$

about $z=z_{1}$. We introduce the quantities $l$ and $\alpha$ defined by the relations

$$
\begin{aligned}
l & =\inf _{f \in \mathcal{F}_{z_{1}}} l_{f}, \\
\alpha & =\sup _{F \in \Omega_{z_{1}}} \alpha_{F},
\end{aligned}
$$

where

$$
l_{f}=\oint_{C}|f(z)| d s
$$

and $s$ is the arc length parameter along $C$. It is clear from the theory of normal families of analytic functions that the word "supremum" may be replaced by "maximum" in (26). It will appear in the course of our work that 
the word "infimum" in (25) may similarly be replaced by "minimum."

We now apply Cauchy's theorem to obtain an inequality between $l$ and $\alpha$. We have

$$
\alpha_{F}=\frac{1}{2 \pi i} \oint_{C} F(z) f(z) d z \quad \leqq \frac{1}{2 \pi} \oint_{C}|f(z)| d s=\frac{l_{f}}{2 \pi} .
$$

Hence for each $F \in \Omega_{z_{1}}$

$$
\alpha_{F} \leqq l / 2 \pi,
$$

and it follows that

$$
\alpha \leqq l / 2 \pi .
$$

We make the conclusion (29) for every $F \in \Omega_{z_{1}}$, and therefore it is necessary to define the integral (28) over $C$ even when $F$ is not continuous in $D+C$. This is done quite easily. For $F$ is bounded and therefore has a nontangential limit almost everywhere on $C[13]$. We select a sequence of contours in $D$ converging to $C$ and we apply the Lebesgue theorem to the sequence of integrals so defined. This process yields the generalized Cauchy formula (28), which we can now use on $C$ by defining $F$ almost everywhere on $C$ by its nontangential limits.

We shall now show that (30) may always be replaced by the equality

$$
\alpha=l / 2 \pi,
$$

and in the process we shall find the extremal functions $f_{0}(z)$ and $F_{0}(z)$ yielding respectively the minimum and maximum values of $l_{f}=\mathscr{S}_{C}|f(z)| d s$ and $\alpha_{F}=F^{\prime}\left(z_{1}\right)$.

Equality would hold in (30) if there existed functions $f_{0} \in \mathcal{F}_{z_{1}}$ and $F_{0} \in \Omega_{z_{1}}$ such that

$$
\frac{1}{2 \pi i} \oint_{C} F_{0}(z) f_{0}(z) d z=\frac{1}{2 \pi} \oint_{C}\left|f_{0}(z)\right| d s .
$$

The integral on the left may also be written

$$
\frac{1}{2 \pi i} \oint_{C} F_{0}(z) f_{0}(z) z^{\prime}(s) d s,
$$

where $z(s)$ is the parametric function describing the curves $C_{1}, \cdots, C_{n}$. Thus (32) will hold if and only if

$$
\frac{F_{0}(z) f_{0}(z) z^{\prime}(s)}{i} \geqq 0, \quad z \in C,
$$

and 


$$
\left|F_{0}(z)\right| \equiv 1 \text {, }
$$

The equation (34) may be rewritten in the more convenient form

$$
\operatorname{Im}\left\{\log \frac{F_{0}(z) f_{0}(z) z^{\prime}(s)}{i}\right\}=0, \pm 2 \pi, \pm 4 \pi, \cdots,
$$

at points $z \in C$ where $f_{0}(z) \neq 0$.

We denote by $G(z ; \zeta)$ the Green's function in $D$; that is, let $G(z ; \zeta)$ be that harmonic function in $D$ with a positive logarithmic singularity at $z=\zeta$ and zero boundary values. We set

$$
\phi^{\prime}(z)=-\frac{\partial G\left(z ; x_{1}\right)}{\partial x}+i \frac{\partial G\left(z ; x_{1}\right)}{\partial y},
$$

where $x_{1}$ is any fixed point of $D$ and $z=x+i y$. We find that

$$
\frac{\phi^{\prime}(z) z^{\prime}(s)}{i}>0, \quad z \in C,
$$

and it follows from the argument principle that $\phi^{\prime}(z)$ has $n-1$ zeros $x_{2}, \cdots, x_{n}$ in $D$. We note that for our purposes any function $\phi^{\prime}(z)$ with the property (38) on $C$ would do as well as the particular one we have chosen. Our choice is merely governed by the fact that Green's function leads us to the simplest calculations. We now arrive at the final form

$$
N(z)=\operatorname{Im}\left\{\log \frac{F_{0}(z) f_{0}(z)}{\phi^{\prime}(z)}\right\}=0, \pm 2 \pi, \pm 4 \pi, \cdots, z \in C_{\mu},
$$

$\mu=1, \cdots, n$, of (36). The function $N(z)$ is harmonic in $D$ except for the zeros, $z_{1}, z_{2}, \cdots, z_{m}$, of $F_{0}(z)$, where it has singularities of the form

$$
N(z)=-\arg \left(z-z_{1}\right)+\cdots, N(z)=\arg \left(z-z_{j}\right)+\cdots, j \geqq 2,
$$

and the points $x_{1}, \cdots, x_{n}$, where it has singularities of the form

$$
N(z)=\arg \left(z-x_{1}\right)+\cdots, N(z)=-\arg \left(z-x_{j}\right)+\cdots, \quad j \geqq 2 .
$$

We make the assumption that $f_{0}(z)$ has no zeros. A harmonic function with the boundary behavior (39) and these singularities will exist only if there are precisely $n$ points $z_{j}$. Thus we require that $m=n$.

If the requirement $m=n$ is fulfilled, then a harmonic function of the type $N(z)$ exists, but it does not necessarily have a single-valued conjugate harmonic function $\bar{N}(z)$. We may add to the function

$$
\operatorname{Im}\left\{\log \frac{\left(z-x_{1}\right) \prod_{\mu=2}^{n}\left(z-z_{\mu}\right)}{\left(z-z_{1}\right) \prod_{\mu=2}^{n}\left(z-x_{\mu}\right)}\right\}
$$


such a harmonic function $u(z)$ that the sum function is of the type $N(z)$. Here $u(z)$ is the solution of a Dirichlet problem with continuous boundary values which are determined up to integral multiples of $2 \pi$. It is convenient to introduce the harmonic functions $\omega_{1}(z), \cdots, \omega_{n}(z)$ in $D$, known as the harmonic measures of the boundary curves $C_{1}, \cdots, C_{n}$. $\dot{\omega}_{\mu}(z)$ is that harmonic function in $D$ with boundary values 1 on $C_{\mu}$ and zero boundary values on the remainder of $C$. It is clear that $u(z)$ is determined up to a linear combination of the $\omega_{\mu}(z)$ with coefficients which are integral multiples of $2 \pi$.

We have now to determine the conditions upon the points $z_{2}, z_{3}, \cdots, z_{n}$ that $F_{0}(z)$ exist in $\Omega_{z_{1}}$ with zeros only at $z_{1}$ and these $n-1$ points and with unit modulus on $C$. This question can be expressed in terms of the Green's function $G(z ; \zeta)$ in $D$. If $F_{0}(z)$ exists, $\log \left|F_{0}(z)\right|$ has the form

$$
\log \left|F_{0}(z)\right|=-\sum_{\mu=1}^{n} G\left(z ; z_{\mu}\right)
$$

Thus the condition that $F_{0}(z)$ exist with the zeros $z_{1}, z_{2}, \cdots, z_{n}$ is that the expression on the right have a conjugate harmonic function whose periods about the $C_{\mu}$ are all $2 \pi, \mu=1, \cdots, n$. This condition we can express by the relations

$$
\sum_{\mu=1}^{n} \omega_{\nu}\left(z_{\mu}\right)=1, \quad \nu=1,2, \cdots, n,
$$

as is well known.

We require further of the $z_{\mu}$ that they be so located that $\bar{N}(z)$, the conjugate harmonic function of $N(z)$, be single-valued. This will be so if the integrals

$$
\tau_{\nu}=\oint_{C \nu} \frac{\partial N}{\partial n} d s
$$

$\nu=1, \cdots, n$,

-vanish, where $\partial / \partial n$ denotes differentiation 'with respect to the inner normal of $C$. To investigate the integrals (45), we introduce cuts $\gamma_{\mu}$ in $D$ joining $x_{\mu}$ to $z_{\mu}$. We then apply Green's theorem to $N(z)$ and $\omega_{\nu}(z)$ in the domain $D^{*}$ bounded by the $C_{\mu}$ and the cuts $\gamma_{\mu}$. We find

$$
\sum_{\mu=1}^{n} \oint_{c_{\mu+\gamma \mu}}\left(\omega_{\nu} \frac{\partial N}{\partial n}-N \frac{\partial \omega_{\nu}}{\partial n}\right) d s=0, \quad \nu=1, \cdots, n,
$$

where the improper integrals over $\gamma_{\mu}$ are to be extended over both sides of these $n$ cuts and are to be taken in the sense of the Cauchy principal value. Denoting by $\bar{\omega}_{\nu}(z)$ the conjugate harmonic function to $\omega_{\nu}(z)$, we obtain

$$
\oint_{\gamma_{1}} N \frac{\partial \omega_{\nu}}{\partial n} d s=2 \pi \int_{x_{1}}^{z_{1}} d \bar{\omega}_{\nu}(z), \quad \nu=1, \cdots, n,
$$




$$
\begin{aligned}
& \oint_{\gamma \mu} N \frac{\partial \omega_{\nu}}{\partial n} d s=-2 \pi \int_{x_{\mu}}^{z_{\mu}} d \bar{\omega}_{\nu}(z), \quad \nu=1, \cdots, n ; \mu=2, \cdots, n, \\
& \oint_{\gamma \mu} \omega_{\nu} \frac{\partial N}{\partial n} d s=0, \quad \mu, \nu=1, \cdots, n \text {, } \\
& \oint_{C_{\mu}} N \frac{\partial \omega_{\nu}}{\partial n} d s=2 m_{\mu} \pi P_{\mu \nu}, \quad \mu, \nu=1, \cdots, n \text {, } \\
& \oint_{C_{\mu}} \omega_{\nu} \frac{\partial N}{\partial n} d s=\left\{\begin{array}{ll}
0, & \nu \neq \mu, \\
\tau_{\nu}, \nu=\mu,
\end{array} \quad \mu, \nu=1, \cdots, n,\right.
\end{aligned}
$$

where the integers $m_{\mu}$ depend on $u(z)$ and

$$
P_{\mu \nu}=\oint_{c_{\mu}} \frac{\partial \omega_{\nu}}{\partial n} d s
$$

We conclude from (46) that we must require

$$
\tau_{\nu}=2 \pi \int_{x_{1}}^{z_{1}} d \bar{\omega}_{\nu}(z)-2 \pi \sum_{\mu=2}^{n} \int_{x_{\mu}}^{z_{\mu}} d \bar{\omega}_{\nu}(z)+2 \pi \sum_{\mu=1}^{n} m_{\mu} P_{\mu \nu}=0, \nu=1, \cdots, n .
$$

If we set $w_{\nu}(z)=\omega_{\nu}(z)+i \bar{\omega}_{\nu}(z)$, we may summarize the conditions (44) and (53) in the form

$$
\begin{aligned}
\sum_{\mu=2}^{n} \int_{x_{\mu}}^{z_{\mu}} w_{\nu}^{\prime}(z) d z=-\int_{x_{1}}^{z_{1}} \overline{w_{\nu}^{\prime}(z) d z}+\sum_{\mu=1}^{n}\left\{\frac{1}{n}-\omega_{\nu}\left(x_{\mu}\right)+i m_{\mu} P_{\mu \nu}\right\} \\
\nu=1, \cdots, n
\end{aligned}
$$

Note that any $n-1$ of the conditions (54) imply the remaining condition, and note that by a proper determination of $u(z)$ we may choose the $m_{\mu}$ to be any integers.

It is well known that to each domain $D$ of finite connectivity $n$ there corresponds a symmetric closed Riemann surface $S$ of genus $p=n-1$. This surface $S$ is defined by taking two replicas of $D$ and identifying the corresponding points of the boundaries $C$. The local parameter for a point $z$ of $S$ which belongs to $C$ is defined by mapping a small sector of $D$ about $z$ upon a semi-circle so that the diameter of the circle corresponds to the points at $C$ near $z$. We then use the variable in the semi-circle as the local parameter of $z$. This parameter is defined on both replicas of $D$ by symmetry. That is, corresponding points of the two replicas are represented by points of the circle which are symmetric in the diameter corresponding to $C$. The surface we have so defined is a Riemann surface by virtue of the Schwarz reflection principle.

On $S$, if we take the curves $C_{2}, \cdots, C_{n}$ to be the canonical cuts $a_{1}, \cdots, a_{p}$ mentioned in Chapter $I$, the harmonic measure functions $w_{2}(z), w_{3}(z), \cdots$, $w_{n}(z)$ give rise to the $(n-1)$ linearly independent normal integrals of the 
first kind $u_{1}=\pi i w_{2} / 2+J_{2}, u_{2}=\pi i w_{3} / 2+J_{3}, \cdots, u_{p}=\pi i w_{n} / 2+J_{n}$. Here the $J_{i}$ are constants to be determined from (7) and (8). The determination of the $z_{\mu}, \mu=2, \cdots, n$, from the equations (54) is upon $S$ merely a special case of the Jacobi inversion problem (3). We have shown in Chapter I how this problem can be solved in terms of Riemann theta-functions.

In our specific case, we know there exists in $D$ a single-valued function $\zeta(z)$ with real boundary values and with $q \geqq n$ poles on $C$. We can continue $\zeta(z)$ analytically by the Schwarz principle of reflection onto $S$, and indeed it was by means of such functions $\zeta(z)$ that Schottky [17] first introduced the Riemann surface $S$ associated with $D$. The Schottky function $\zeta(z)$ maps $S$ upon a covering surface of the $\zeta$-plane of $q$ sheets, and we have now merely to determine the points $\zeta_{\mu}=\zeta\left(z_{\mu}\right)$ by means of the formula (6). It suffices to set

$$
\begin{aligned}
V_{\nu-1}= & \frac{\pi i}{2}-\frac{\pi i \omega_{\nu}\left(x_{1}\right)}{2}-\frac{\pi i}{2} \int_{x_{1}}^{z_{1}} d \bar{\omega}_{\nu}-\sum_{\mu=1}^{n} \frac{\pi m_{\mu} P_{\mu \nu}}{2} \\
& -\sum_{\mu=2}^{n} \frac{\pi \bar{\omega}_{\nu}\left(x_{\mu}\right)}{2}+\sum_{\mu=2}^{n} J_{\mu}, \quad \nu=2, \cdots, n .
\end{aligned}
$$

The constants $J_{2}, \cdots, J_{n}$ are determined from (7). We see that the points $z_{2}, \cdots, z_{n}$ are analytic functions of $\bar{z}_{1}$. Note that $b_{\mu \nu}=\pi P_{\mu \nu} / 2$.

Thus we have shown that on $S$ there exist $n-1$ points $z_{\mu}$ and corresponding paths of integration from $x_{\mu}$ to $z_{\mu}$ such that (54) is fulfilled. We need to show that the $z_{\mu}$ all lie in the "upper" half $D$ of $S$. Suppose that $z_{1}, z_{2}, \cdots, z_{r}$ lie in $D$, but that $z_{r+1}, \cdots, z_{n}$ do not. Let $z_{r+1}^{*}, \cdots, z_{n}^{*}$ be the points in $D$ symmetric to $z_{r+1}, \cdots, z_{n}$. Then

$$
\begin{aligned}
\sum_{\mu=1}^{r} \omega_{\nu}\left(z_{\mu}\right)-\sum_{\mu=r+1}^{n} \omega_{\nu}\left(z_{\mu}^{*}\right)=\sum_{\mu=1}^{r} \omega_{\nu}\left(z_{\mu}\right)+\sum_{\mu=r+1}^{n} \omega_{\nu}\left(z_{\mu}\right)+2 k_{\nu}=1 & +2 k_{\nu} \\
\nu & =1, \cdots, n,
\end{aligned}
$$

where the $k_{\nu}$ are integers. We add the absolute values of the expressions (56) to obtain

$$
\sum_{\nu=1}^{n}\left|\sum_{\mu=1}^{r} \omega_{\nu}\left(z_{\mu}\right)-\sum_{\mu=r+1}^{n} \omega_{\nu}\left(z_{\mu}^{*}\right)\right|=\sum_{\nu=1}^{n}\left|1+2 k_{\nu}\right| \geqq n .
$$

But

$$
\sum_{n=1}^{n}\left\{\sum_{\mu=1}^{r} \omega_{\nu}\left(z_{\mu}\right)+\sum_{\mu=r+1}^{n} \omega_{\nu}\left(z_{\mu}^{*}\right)\right\}=n,
$$

and thus, since $\omega_{\nu}(z)>0$ for $z \in D$, the inequality (57) can only hold if all terms within each absolute value sign are of one sign. Thus either $r=0$ or $r=n$. But $r \geqq 1$, and therefore $r=n$. Thus all $z_{\mu}$ either lie in $D$ or on $C$. Now if $z_{j}$, say, lies on $C_{l}$ we have by (54) 


$$
\sum_{\mu=1, \mu \nLeftarrow j}^{n} \omega_{l}\left(z_{\mu}\right)=1-\omega_{l}\left(z_{j}\right)=0,
$$

and since $\omega_{l}(z)>0, z \in D$, we must have $z_{k} \in C, k=1, \cdots, n$. But $z_{1} \notin C$, and therefore we arrive at a contradiction. Thus all $z_{\mu}$ lie in $D$.

The one remaining difficulty concerns the homology type of the integration paths from the $x_{\mu}$ to the $z_{\mu}$. If we integrate from $x_{\mu}$ to $z_{\mu}$ along a path in $D$, we find that

$$
\sum_{\mu=1}^{n} \omega_{\nu}\left(z_{\mu}\right)=1+2 k_{\nu}, \quad \nu=1, \cdots, n,
$$

and clearly $k_{\nu} \geqq 0$, since the left-hand sides are positive. Addition of these equations shows that $k_{\nu}=0, \nu=1, \cdots, n$. We now choose the $m_{\mu}$, upon which no condition has been imposed until now, so that (54) holds with the paths of integration in $D$ which we have selected. Thus the Jacobi determination of the $z_{\mu}$ will give us the solution of our problem.

Having determined the $z_{\mu}$, we set up a function $F_{0}(z)$ with the zeros $z_{1}$, $z_{2}, \cdots, z_{n}$, find the analytic function $N(z)+i \bar{N}(z)$ by solving the boundary value problem (39), and solve for $f_{0}(z)$ to obtain

$$
f_{0}(z)=\frac{\phi^{\prime}(z) \exp (-\bar{N}(z)+i N(z))}{F_{0}(z)} .
$$

We can now see, using (28) and (32), that

$$
\alpha_{F} \leqq l_{f_{0}} / 2 \pi,
$$$$
F \in \Omega_{z_{1}},
$$

with equality holding for and only for $F_{0}$, and

$$
\alpha_{F_{0}} \leqq l_{f} / 2 \pi \text {, }
$$

with equality holding for and only for $f_{0}$. The uniqueness of $F_{0}$ follows because it is uniquely determined in modulus and argument on $C$, and the uniqueness of $f_{0}$ follows from that of $F_{0}$ by (61).

It is necessary to make a few remarks about uniqueness in the case where $F$ is merely defined almost everywhere on $C$ by radial limits. Our proof has shown only that these radial limits are uniquely determined almost everywhere on $C$. However, it is a consequence of a well known theorem of the Riesz brothers [13] that the radial limits of $F$ on a set of $C$ of positive measure determine $F$ completely. Hence our proof covers this case.

From the $(n-1)$-parameter family of maps $F \in \Omega_{z_{1}}$ covering the unit circle $n$ times we have now a more or less explicit method of selecting $F_{0}$ by solving the Jacobi problem (54) in terms of Riemann theta-functions. Furthermore, when $D$ can be mapped on the $n$-times-covered unit circle with symmetry in the real axis, then we can say that this symmetric mapping is 
$F_{0}$. In the case $n=2$ a symmetric mapping on circles can always be found, and thus we obtain the extension of Schwarz's lemma here by elementary methods and without the use of the Jacobi inversion problem.

This property of the symmetric mapping $F_{0}$ is proved as follows. We suppose that the original domain $D$ and the image domain by $F_{0}$ are both symmetric in the real axis, we suppose that all the contours $C_{\nu}$ intersect the real axis, and we suppose that $z_{1}, \cdots, z_{n}$ lie on the real axis and are separated from one another there by the contours $C_{\nu}$. We have

$$
F_{0}(\bar{z})=\overrightarrow{F_{0}(z)} \text {. }
$$

We choose $x_{1}$ to lie on the same segment of the real axis in $D$ as $z_{1}$. The Green's function $G\left(z ; x_{1}\right)$ is symmetric, and it is readily verified that

$$
N(\bar{z})+i \bar{N}(\bar{z})=-N(z)+i \bar{N}(z) .
$$

Hence

$$
\tau_{\nu}=\oint_{c_{\nu}} \frac{\partial N}{\partial n} d s=-\oint_{c_{\nu}} \frac{\partial N}{\partial n} d s=-\tau_{\nu}, \quad . \nu=1, \cdots, n,
$$

and therefore $\tau_{\nu}=0, \nu=1, \cdots, n$. Thus $\bar{N}(z)$ has no periods about the $C_{\nu}$, which is all that we require.

We summarize the principal results of this chapter in a theorem.

THEOREM 1. Let $f_{0}(z)$ and $F_{0}(z)$ be extremal functions for the problems (25) and (26). Such extremal functions exist and are uniquely determined. The extremal quantities $l$ and $\alpha$ satisfy (31). The functions $f_{0}, F_{0}$ satisfy (34), (43), and (61), and the zeros $z_{2}, \cdots, z_{n}$ of $F_{0}$ solve the Jacobi inversion problem (54).

IV. The Szegö kernel function. Consider now the function

$$
K\left(z, z_{1}\right)=\frac{F_{0}(z)\left(f_{0}(z)\right)^{1 / 2}}{2 \pi}
$$

where $\left(f_{0}(z)\right)^{1 / 2}$ is chosen so that $\left(z-z_{1}\right)\left(f_{0}(z)\right)^{1 / 2} \rightarrow 1$ as $z \rightarrow z_{1}$. This function is single-valued in $D$, since $f_{0}$ has no zeros and only a double pole there, and since the variation of the argument of $f_{0}$ on each $C_{\mu}$ is either zero or $-4 \pi$, by (34). We find for every element $\psi(z)$ of the class $R$ of functions regular in $D$ and continuous in $D+C$ the relation

$$
\psi\left(z_{1}\right)=\oint_{C} \psi(z) \overline{K\left(z, z_{1}\right)} d s .
$$

Indeed, we have by (34), (35), and (67)

$$
\oint_{c} \psi(z) \overline{K\left(z, z_{1}\right)} d s=\frac{1}{2 \pi} \oint_{C} \psi(z) \overrightarrow{F_{0}(z)\left(f_{0}(z)\right)^{1 / 2} d s}
$$




$$
\begin{aligned}
& =\frac{\overline{i^{1 / 2}}}{2 \pi} \oint_{C} \psi\left(\frac{\overline{f_{0} F_{0} z^{\prime}}}{i}\right)^{1 / 2} \overline{F_{0}^{1 / 2}}\left(z^{\prime}\right)^{1 / 2} d s \\
& =\frac{\overline{i^{1 / 2}}}{2 \pi} \oint_{C} \psi\left(\frac{f_{0} F_{0} z^{\prime}}{i}\right)^{1 / 2} \overline{F_{0}^{1 / 2}}\left(z^{\prime}\right)^{1 / 2} d s \\
& =\frac{1}{2 \pi i} \oint_{C} \psi(z)\left(f_{0}(z)\right)^{1 / 2} d z .
\end{aligned}
$$

But by the residue theorem we have

$$
\frac{1}{2 \pi i} \oint_{\dot{c}} \psi(z)\left(f_{0}(z)\right)^{1 / 2} d z=\psi\left(z_{1}\right)
$$

Hence formula (68) is proved.

We can now state that among all functions $\psi(z) \in R$ with $\psi\left(z_{1}\right)=1$, the function

$$
K\left(z, z_{1}\right) / K\left(z_{1}, z_{1}\right)
$$

yields the minimum $L$ of the integral

$$
L_{\psi}=\oint_{C}|\psi(z)|^{2} d s
$$

In fact, we have by Schwarz's inequality

$$
\begin{aligned}
1=\psi\left(z_{1}\right)^{2} & =\left\{\oint_{c} \psi(z) \overline{K\left(z, z_{1}\right)} d s\right\}^{2} \\
& \leqq \oint_{c} K\left(z, z_{1}\right) \overline{K\left(z, z_{1}\right)} d s \cdot \oint_{C}|\psi(z)|^{2} d s \\
& =K\left(z_{1}, z_{1}\right) \oint_{C}|\psi(z)|^{2} d s .
\end{aligned}
$$

Therefore

$$
\oint_{c}|\psi(z)|^{2} d s \geqq \frac{1}{K\left(z_{1}, z_{1}\right)}=L,
$$

and equality holds only for the function (71).

For the study of the indefinite integral of the function $f_{0}(z)$ it is convenient to know that its residue $a_{-1}$ at the pole at $z=z_{1}$ vanishes. We have by (34) and (35)

$$
\overline{a_{-1}}=\overline{\frac{1}{2 \pi i} \oint_{c} f_{0}(z) d z}=\frac{1}{2 \pi} \oint_{c} \overline{\left[\frac{f_{0} F_{0} z^{\prime}}{i}\right]} F_{0} d s \quad=\frac{1}{2 \pi i} \oint_{c} f_{0} F_{0}^{2} d z=0
$$


This relation implies, incidentally, the obvious fact that $F_{0}$ maximizes $\left|F^{\prime}\left(z_{1}\right)\right|$ among all functions $F(z)$ in $D$ with $|F(z)| \leqq 1$ there, regardless of where $F\left(z_{1}\right)$ lies in the unit circle.

We define the functions

$$
\begin{aligned}
& M\left(z, z_{1}\right)=4 \pi^{2} \int^{z} K\left(\zeta, z_{1}\right)^{2} d \zeta \\
& N\left(z, z_{1}\right)=-\int^{z} f_{0}(\zeta) d \zeta
\end{aligned}
$$

which have periods about the contours $C_{\mu}$. We find that

$$
\begin{aligned}
M^{\prime}\left(z, z_{1}\right) z^{\prime} & =4 \pi^{2} K\left(z, z_{1}\right)^{2} z^{\prime} \\
& =F_{0}^{2} f_{0} z^{\prime} \\
& =F_{0} i \frac{F_{0} f_{0} z^{\prime}}{i} \\
& =F_{0} i \overline{\left.\frac{F_{0} f_{0} z^{\prime}}{i}\right]} \\
& =-\overline{f_{0}(z) z^{\prime}}=\overline{N^{\prime}\left(z, z_{1}\right) z^{\prime}}
\end{aligned}
$$

for $z \in C$. Thus the multiple-valued functions

$$
\begin{aligned}
& \Phi\left(z, z_{1}\right)=N\left(z, z_{1}\right)+M\left(z, z_{1}\right), \\
& \Psi\left(z, z_{1}\right)=N\left(z, z_{1}\right)-M\left(z, z_{1}\right)
\end{aligned}
$$

have constant imaginary or real part, respectively, on each $C_{\mu} . \Phi$ and $\Psi$ are therefore multiple-valued Schottky functions and they may be expressed in terms of the derivatives of Green's function and the harmonic measures.

Indeed, $\Phi$ and $\Psi$ each have just one pole at $z_{1}$, and the residue in both cases is 1 . We set $z_{1}=x_{1}+i y_{1}, z=x+i y$ and define

$$
\begin{gathered}
\frac{\partial}{\partial z}=\frac{1}{2}\left(\frac{\partial}{\partial x}-i \frac{\partial}{\partial y}\right), \quad \frac{\partial}{\partial z_{1}}=\frac{1}{2}\left(\frac{\partial}{\partial x_{1}}-i \frac{\partial}{\partial y_{1}}\right), \\
\frac{\partial}{\partial \bar{z}_{1}}=\frac{1}{2}\left(\frac{\partial}{\partial x_{1}}+i \frac{\partial}{\partial y_{1}}\right) .
\end{gathered}
$$

Now let $H(z ; \zeta)$ be the conjugate harmonic function of the Green's function $G(z ; \zeta)$ and let

$$
\phi(z ; \zeta)=G(z ; \zeta)+i H(z ; \zeta)
$$

Then $\partial \phi\left(z ; z_{1}\right) / \partial x_{1}$ has a simple pole of residue 1 at $z_{1}$ and has imaginary boundary values. Likewise, $-i \partial \phi\left(z ; z_{1}\right) / \partial y_{1}$ has a simple pole of residue 1 at $z_{1}$ and has real boundary values. Hence $\Phi+i \partial \phi / \partial y_{1}$ and $\Psi-\partial \phi / \partial x_{1}$ may be 
expressed as linear combinations of the functions $w_{\nu}(z)$ with pure imaginary and real coefficients, respectively. It follows that $N-\partial \phi / \partial z_{1}$ and $M+\partial \phi / \partial \bar{z}_{1}$ are expressible in terms of the $w_{\nu}(z)$. Applying the operator $\partial / \partial z$, we verify the explicit formulas( $\left.{ }^{4}\right)$

$$
\begin{gathered}
M^{\prime}\left(z, z_{1}\right)=\frac{-2}{\left\|w_{\nu}^{\prime}\left(z_{\mu}\right)\right\|}\left\|\begin{array}{ll}
\frac{\partial^{2} G\left(z ; z_{1}\right)}{\partial z \partial \bar{z}_{1}} & w_{\nu}^{\prime}(z) \\
\frac{\partial^{2} G\left(z_{\mu} ; z_{1}\right)}{\partial z_{\mu} \partial \bar{z}_{1}} & w_{\nu}^{\prime}\left(z_{\mu}\right)
\end{array}\right\|, \\
N^{\prime}\left(z, z_{1}\right)=\frac{2}{\left\|\overline{w_{\nu}^{\prime}\left(z_{\mu}\right)}\right\|}\left\|\begin{array}{ll}
\frac{\partial^{2} G\left(z ; z_{1}\right)}{\partial z \partial z_{1}} & w_{\nu}^{\prime}(z) \\
\frac{\partial^{2} G\left(z_{\mu} ; z_{1}\right)}{\partial \bar{z}_{\mu} \partial z_{1}} & \overline{w_{\nu}^{\prime}\left(z_{\mu}\right)}
\end{array}\right\|,
\end{gathered}
$$

where the indices $\nu$ and $\mu$ run from 2 to $n$. Here we carry out the verification by constructing the coefficients of the $w_{\nu}^{\prime}(z)$ in the formula for $M^{\prime}\left(z, z_{1}\right)$ so that $M^{\prime}\left(z_{\mu}, z_{1}\right)=0, \mu=2, \cdots, n$. The formula for $N^{\prime}\left(z, z_{1}\right)$ is then obtained by using the negative conjugates of these coefficients, as we see from our discussion of $\Phi$ and $\Psi$. We must assume that

$$
\left\|w_{r}^{\prime}\left(z_{\mu}\right)\right\| \neq 0
$$

for the formulas (83) and (84) to be valid, and when this is not the case our representation becomes somewhat more complicated.

Inspection of (76) and (77) shows that

$$
F_{0}(z)=\left(-\frac{M^{\prime}\left(z, z_{1}\right)}{N^{\prime}\left(z, z_{1}\right)}\right)^{1 / 2} \text {. }
$$

Thus our extremal functions may all be expressed in terms of Green's function and the harmonic measures. Note that from the property (78) alone we can conclude that

$$
M^{\prime}\left(z, z_{1}\right) / N^{\prime}\left(z, z_{1}\right)
$$

maps $D$ upon the unit circle covered $2 n$ times. We derive further from (86) the interesting relation

$$
\alpha=2 \pi K\left(z_{1}, z_{1}\right)=2 \pi / L,
$$

whence by (31) we have

$$
l L=4 \pi^{2}
$$

(4) In this paper we use for determinants the notation customarily employed for matrices in order to avoid confusion with the notation for absolute value. 
It is well known that there exist complete systems $\left\{\psi_{\nu}(z)\right\}$ of polynomials and rational functions regular in $D$ by means of which every function $\psi(z) \in R$ can be approximated uniformly in each closed subdomain of $D$ [20]. We remark that any complete system each of whose elements is analytic in a domain $\tilde{D}$ containing $D+C$ would serve us just as well as the polynomials and rational functions. We assume that such a system has been orthonormalized over $C$ so that

$$
\oint_{C} \psi_{\nu}(z) \overline{\psi_{\mu}(z)} d s=\delta_{\nu \mu}=\left\{\begin{array}{l}
1, \nu=\mu, \\
0, \nu \neq \mu .
\end{array}\right.
$$

Szegö [19] has shown that among all functions

$$
\psi(z)=\sum_{k=1}^{m} a_{k} \psi_{k}(z)
$$

with

$$
\psi\left(z_{1}\right)=1
$$

and $m$ fixed, the function

$$
K_{m}\left(z, z_{1}\right) / K_{m}\left(z_{1}, z_{1}\right)
$$

gives the minimum value $K_{m}\left(z_{1}, z_{1}\right)^{-1}$ to the integral

$$
\oint_{C}|\psi(z)|^{2} d s
$$

where

$$
K_{m}\left(z, z_{1}\right)=\sum_{k=1}^{m} \psi_{k}(z) \overline{\psi_{k}\left(z_{1}\right)}
$$

From this property he concludes that $K_{m}\left(z, z_{1}\right)$ converges uniformly in every closed subdomain of $D$ to a function $K_{S}\left(z, z_{1}\right)$, the Szegö kernel function of $D$. Szegö treats only the case of a simply-connected domain $D$, but the generalization of his proofs is in part readily made for multiply-connected domains. For the details of this technique, see also Bergman [3].

We shall prove somewhat differently the convergence of the sequence $K_{m}\left(z, z_{1}\right)$, and we shall show that the limit function $K_{S}\left(z, z_{1}\right)$ is given by

$$
K_{S}\left(z, z_{1}\right)=K\left(z, z_{1}\right) \text {. }
$$

Indeed, we have shown that $K\left(z, z_{1}\right) K\left(z_{1}, z_{1}\right)^{-1}$ minimizes (94) among all functions $\psi(z) \in R$ satisfying (92) and we have shown that the minimum value is

$$
L=1 / K\left(z_{1}, z_{1}\right)
$$


Since the $C_{\mu}$ are analytic curves, and since $K\left(z, z_{1}\right)$ is analytic across them, we can approximate $K\left(z, z_{1}\right)$ uniformly in $D+C$ by linear combinations of the functions $\psi_{\nu}(z)$. Thus we see that the minimum value $K_{m}\left(z_{1}, z_{1}\right)^{-1}$ of (94) for functions (91) tends to $K\left(z_{1}, z_{1}\right)^{-1}$ as $m$ tends to infinity, or

$$
\lim _{m \rightarrow \infty} K_{m}\left(z_{1}, z_{1}\right)=K\left(z_{1}, z_{1}\right) \text {. }
$$

From (68) we find, since $K_{m}\left(z_{1}, z_{1}\right)$ is real, that

$$
\begin{aligned}
\oint_{C}\left|K\left(z, z_{1}\right)-K_{m}\left(z, z_{1}\right)\right|^{2} d s & =K\left(z_{1}, z_{1}\right)+K_{m}\left(z_{1}, z_{1}\right)-2 \operatorname{Re}\left\{K_{m}\left(z_{1}, z_{1}\right)\right\} \\
& =K\left(z_{1}, z_{1}\right)-K_{m}\left(z_{1}, z_{1}\right)=\epsilon_{m}
\end{aligned}
$$

where $\epsilon_{m} \rightarrow 0$ as $m \rightarrow \infty$. We conclude that

$$
\left|K\left(t, z_{1}\right)-K_{m}\left(t, z_{1}\right)\right|^{2} \leqq \epsilon_{m} K(t, t),
$$

by (73), and this proves our statement. Here $K(z, t)$ is the function (67) with normalization point at $t \in D$ rather than at $z_{1} \in D$.

The result may be expressed in the form

$$
K\left(z, z_{1}\right)=\sum_{k=1}^{\infty} \psi_{k}(z) \overline{\psi_{k}\left(z_{1}\right)}
$$

We have shown that the Szegö kernel function $K_{S}(z, z)$ is independent of the orthonormal system $\left\{\psi_{\nu}(z)\right\}$ and that it may be expressed in terms of Green's function and the harmonic measures. In fact, from (83) and (101) we obtain

$$
K_{S}\left(z, z_{1}\right)^{2}=-\frac{1}{2 \pi^{2}} \frac{1}{\left\|w_{\nu}^{\prime}\left(z_{\mu}\right)\right\|}\left\|\begin{array}{ll}
\frac{\partial^{2} G\left(z ; z_{1}\right)}{\partial z \partial \bar{z}_{1}} & w_{\nu}^{\prime}(z) \\
\frac{\partial^{2} G\left(z_{\mu} ; z_{1}\right)}{\partial z_{\mu} \partial \bar{z}_{1}} & w_{\nu}^{\prime}\left(z_{\mu}\right)
\end{array}\right\| .
$$

Schiffer [16] has shown that the Bergman kernel function $K_{B}\left(z, z_{1}\right)$ is given by

$$
K_{B}\left(z, z_{1}\right)=-\frac{2}{\pi} \frac{\partial^{2} G\left(z ; z_{1}\right)}{\partial z \partial \bar{z}_{1}} .
$$

Hence the square of the Szegö kernel function is closely related to the Bergman kernel function in multiply-connected as well as in simply-connected domains.

A further consequence of our investigation follows from (88). We have

$$
\alpha=2 \pi \sum_{k=1}^{\infty}\left|\psi_{k}\left(z_{1}\right)\right|^{2} .
$$

The right-hand side of this equation is readily computed numerically for a 
given domain, and hence we have a method of obtaining the upper bound in Schwarz's lemma in specific cases.

As a final note on kernel functions, we remark that if we add to the class $R$ of functions $\psi(z)$ regular in $D$ the functions with a simple pole at $z=z_{1}$, and if we construct a kernel function $K^{+}\left(z, z_{1}\right)$ for the extended class $R_{1}$ by means of a complete orthonormal system $\left\{\ddot{\psi}_{\nu}(z)\right\}$, we obtain, for $z_{0} \neq z_{1}$,

$$
\begin{aligned}
K^{+}\left(z, z_{0}\right) & =\sum_{k=1}^{\infty} \ddot{\psi}_{k}\left(\overline{z)} \overline{\ddot{\psi}_{k}\left(z_{0}\right)}\right. \\
& =K\left(z, z_{0}\right)+\frac{1}{l}\left(f_{0}(z) \overline{\left.f_{0}\left(z_{0}\right)\right)^{1 / 2}} .\right.
\end{aligned}
$$

Thus we find a kernel function construction of $f_{0}(z)$. And (105) is proved by showing that $f_{0}^{1 / 2}$ is orthogonal to every function $\psi \in R$, so that adjunction of $\left(f_{0} / l\right)^{1 / 2}$ to the system $\left\{\psi_{\nu}\right\}$ complete for $R$ yields a system $\left\{\ddot{\psi}_{\nu}\right\}$ complete for $R_{1}$. We have

$$
\begin{aligned}
\oint_{C} \psi(z) \overline{\left(f_{0}(z)\right)^{1 / 2}} d s & =\overline{i^{1 / 2}} \oint_{c} \psi \overline{\left(\frac{f_{0} F_{0} z^{\prime}}{i}\right)^{1 / 2}}\left(F_{0}\right)^{1 / 2}\left(z^{\prime}\right)^{1 / 2} d s \\
& =\frac{1}{i} \oint_{C} \psi(z)\left(f_{0}(z)\right)^{1 / 2} F_{0}(z) d z=0 .
\end{aligned}
$$

Combining (67), (89), and (105) we obtain

$$
F_{0}(z)=\frac{1}{2 \pi K\left(z_{1}, z_{1}\right)} \frac{K\left(z, z_{1}\right)}{K^{+}\left(z, z_{0}\right)-K\left(z, z_{0}\right)} \overline{\left(f_{0}\left(z_{0}\right)\right)^{1 / 2}},
$$

which expresses $F_{0}(z)$ in terms of kernel functions up to a constant factor.

By contour integration we derive a property of the extremal functions $M^{\prime}\left(z, z_{1}\right)$ and $N^{\prime}\left(z, z_{1}\right)$. We have

$$
\begin{aligned}
0 & =\oint_{C}\left(M^{\prime}\left(z, z_{1}\right)\right)^{1 / 2}\left(M^{\prime}\left(z, z_{0}\right)\right)^{1 / 2} d z \\
& =\oint_{C}\left(M^{\prime}\left(z, z_{1}\right) z^{\prime}\right)^{1 / 2}\left(M^{\prime}\left(z, z_{0}\right) z^{\prime}\right)^{1 / 2} d s \\
& =\oint_{C}\left(N^{\prime}\left(z, z_{1}\right) z^{\prime}\right)^{1 / 2}\left(N^{\prime}\left(z, z_{0}\right) z^{\prime}\right)^{1 / 2} d s \\
& =\frac{2 \pi i\left[i\left(N^{\prime}\left(z_{0}, z_{1}\right)\right)^{1 / 2}+i\left(N^{\prime}\left(z_{1}, z_{0}\right)\right)^{1 / 2}\right]}{2 \pi}
\end{aligned}
$$

whence

$$
N^{\prime}\left(z_{1}, z_{0}\right)=N^{\prime}\left(z_{0}, z_{1}\right) .
$$

Either by considering the integral 


$$
\oint_{C}\left(M^{\prime}\left(z, z_{1}\right)\right)^{1 / 2}\left(N^{\prime}\left(z, z_{0}\right)\right)^{1 / 2} d z
$$

or by using (101) we can also show that

$$
M^{\prime}\left(z_{1}, z_{0}\right)=\overline{M^{\prime}\left(z_{0}, z_{1}\right)} .
$$

In particular, we find that $N^{\prime}\left(z_{1}, z_{0}\right)$ is analytic in $z_{0}$, while $M^{\prime}\left(z_{1}, z_{0}\right)$ is anti-analytic in $z_{0}$. By the identity $K\left(z_{\mu}, z_{1}\right) \equiv 0$ we obtain a second proof that $z_{\mu}$ is an anti-analytic function of $z_{1}, \mu \geqq 2$. The Jacobi inversion problem (54) yielded the first proof.

Ahlfors [1] has shown by variational methods that there exists a harmonic function

$$
h(z)=-G\left(z ; z_{1}\right)+\sum_{\mu=1}^{n} \lambda_{\mu} \omega_{\mu}(z)
$$

with $\partial h / \partial n<0$ on $C$ whose critical points are precisely the zeros $z_{2}, z_{3}, \cdots, z_{n}$ of $F_{0}(z)$. We define an analytic function $p(z)$ by the relation

$$
\operatorname{Re}\{p(z)\}=h(z),
$$

and we find

$$
p^{\prime}(z)=\frac{1}{z-z_{1}}+b_{0}+b_{0}\left(z-z_{1}\right)+\cdots
$$

near $z=z_{1}$ in $D$ and

$$
i p^{\prime}(z) z^{\prime}=\partial h / \partial n<0
$$

on $C$. Now the function

$$
\alpha \frac{p^{\prime}(z)}{F_{0}(z)}=\frac{1}{\left(z-z_{1}\right)^{2}}+\frac{d_{-1}}{\left(z-z_{1}\right)}+d_{0}+d_{1}\left(z-z_{1}\right)+\cdots
$$

is regular in $D$ except at $z=z_{1}$, since the critical points of $p$ are the zeros $z_{2}, \cdots, z_{n}$ of $F_{0}$. From (115) we find

$$
\frac{\alpha p^{\prime}(z)}{F_{0}(z)} \frac{F_{0}(z) z^{\prime}}{i}>0, \quad z \in C,
$$

and comparison with (34) yields the identity

$$
f_{0}(z)=\alpha p^{\prime}(z) / F_{0}(z) \text {. }
$$

Hence also

$$
K\left(z, z_{1}\right)^{2}=\frac{\alpha}{4 \pi^{2}} F_{0}(z) p^{\prime}(z)
$$


We shall investigate these ideas and differentials of the type $p^{\prime}(z) d z$ further in Chapter VI.

We have now the following theorem.

THEOREM 2. In the notation of Theorem 1 let (67) hold. Then for every $\psi \in R$ we have (68) and the function $K\left(z, z_{1}\right) K\left(z_{1}, z_{1}\right)^{-1}$ yields the minimum $L$ of the integral (72) among all functions $\psi \in R$ with $\psi\left(z_{1}\right)=1$. (101) holds, where the expression on the right is the Szegö kernel function of a complete system $\left\{\psi_{k}\right\}$ orthonormalized according to (90). The identities (88) and (89) hold. All our extremal functions can be related to Green's function and the harmonic measures by identities of the form (76), (77), (83), (84), (86), and (105).

V. The problem of Painlevé. We are now in a position to attack the problem of Painlevé. We suppose given a compact set $E$, and we ask for necessary and sufficient conditions upon $E$ that there exist a nonconstant bounded analytic function in the domain $D$ complementary to $E$ which contains the point at infinity. The analogous problem for the existence of bounded harmonic functions has been solved by use of the notion of capacity and by the introduction of Tschebyscheff polynomials (see Nevanlinna [13]).

We choose a sequence of domains $D_{n}$ contained in $D$ and bounded by analytic curves $C^{(n)}$ such that $D_{n}$ converges to $D$. By the convergence we mean that any closed subdomain of $D$ is contained in all $D_{n}$ for all $n$ greater than a sufficiently large $n_{0}$. We might choose the $C^{(n)}$ to be a suitable set of level curves of the Green's functions $G_{n}(z ; \infty)$ of polygonal domains $\tilde{D}_{n}$ exhausting $D$ from within.

We let $\alpha_{n}$ be the quantity (26) for the domain $D_{n}$ and for a point $z_{1}$ contained in all the $D_{n}$. Then it is clear from the theory of normal families of analytic functions [1] that a necessary and sufficient condition that there exist a non-constant bounded analytic function in $D$ is

$$
\alpha=\lim _{n \rightarrow \infty} \alpha_{n}>0 .
$$

This is really a mere restatement of the problem of Painlevé, but we shall transform it by means of our previous results to obtain an effective necessary and sufficient condition.

We point out that for an infinite domain we require of all functions $f \in \mathcal{F}_{z_{1}}$ that they have expansions

$$
f(z)=\frac{a_{2}}{z^{2}}+\frac{a_{3}}{z^{3}}+\cdots
$$

about the point at infinity. This additional requirement results from the fact that $f_{0}(z) d z$ is an invariant differential with respect to conformal mappings. Similarly, $K\left(z, z_{1}\right)^{2} d z$ is an invariant differential, so that we have near infinity 


$$
K\left(z, z_{1}\right)=\frac{c_{1}}{z}+\frac{c_{2}}{z^{2}}+\cdots .
$$

Hence the functions of class $R$ in infinite domains, such as the $D_{n}$, are required to vanish at infinity. This change of normalization, resulting as it does from the invariance of the differentials $f_{0}(z) d z$ and $K\left(z, z_{1}\right)^{2} d z$, does not alter the validity of the results of Chapters III and IV for infinite domains.

Let us prove a property of monotonicity of the functions $l$ and $L$ defined by (25) and (74). If $D_{1}$ is any domain of finite connectivity contained in any other domain $D_{2}$ of finite connectivity, then we see immediately that

$$
\alpha_{1} \geqq \alpha_{2},
$$

with strong inequality holding unless $D_{1}=D_{2}$. Here we assume that our domains both contain the point $z_{1}$ at which $\alpha_{1}$ and $\alpha_{2}$ are evaluated. Denoting by the subscripts 1,2 quantities associated with $D_{1}, D_{2}$, respectively, we obtain from (31) and (88) the striking inequalities

$$
\begin{gathered}
l_{1} \geqq l_{2}, \\
L_{1} \leqq L_{2} .
\end{gathered}
$$

It follows that

$$
K^{(1)}\left(z_{1}, z_{1}\right) \geqq K^{(2)}\left(z_{1}, z_{1}\right),
$$

where now the superscripts indicate the domain of definition of the symbol. The analogue of this inequality is proved directly for the Bergman kernel function, and is one of the most powerful tools for the investigation of conformal mapping by Bergman's methods. But for the Szegö kernel function and for extremal problems with the mean modulus of analytic functions over the boundary of the domain it becomes a much less obvious result.

We return to the problem of Painlevé. We may reword our necessary and sufficient condition by means of the formula (88), or by means of the formula (31). We find that a nonconstant bounded analytic function exists in $D$ if and only if

$$
\lim _{n \rightarrow \infty} K^{(n)}\left(z_{1}, z_{1}\right)>0,
$$

where $K^{(n)}$ is the Szegö kernel function of $D_{n}$. The limit exists by the monotonicity theorem we have just proved. Another necessary and sufficient condition is

$$
\lim _{n \rightarrow \infty} l_{n}>0 \text {. }
$$

We may now draw a conclusion from the first condition (127). We have shown that if there exists a sequence $C^{(n)}$ of curves converging to $E$ and a nonconstant function $\psi$ vanishing at infinity in $D$ with uniformly bounded 
mean modulus

$$
L_{\psi}^{(n)}=\oint_{C^{(n)}}|\psi(z)|^{2} d s
$$

over the $C^{(n)}$, then there exists a bounded analytic function in $D$ not a constant. If the $C^{(n)}$ can be chosen to have uniformly bounded lengths, then this condition,

$$
\limsup _{n \rightarrow \infty} L_{\psi}^{(n)}<\infty,
$$

is considerably weaker than the condition of boundedness in $D$. We obtain from (88) the inequality

$$
\alpha=\lim _{n \rightarrow \infty} \alpha_{n} \geqq \frac{2 \pi}{\limsup _{n \rightarrow \infty} L_{\psi}^{(n)}} .
$$

In connection with the condition (128) we remark that if $z_{1}=\infty$ the normalization condition (23) for the class $\mathcal{F}_{z_{1}}=\mathcal{F}_{\infty}$ becomes

$$
f(z)=1+\frac{a_{1}}{z}+\frac{a_{2}}{z^{2}}+\cdots,
$$

since $f_{0} d z$ is a differential. We find in consequence of this that, if $\alpha_{\infty}^{(n)}$ denotes the quantity (26) for $z_{1}=\infty$,

$$
K_{(n)}^{+}(z, \infty)=\left(f_{0}^{(n)}\right)^{1 / 2} / 2 \pi \alpha_{\infty}^{(n)}
$$

is the Szegö kernel function of the class of all analytic functions in $D_{n}$ with normalization point at infinity. The proof is quite analogous to the similar proofs carried out in Chapter IV. The condition (128) now reads

$$
\lim _{n \rightarrow \infty} K_{(n)}^{+}(\infty, \infty)<\infty .
$$

We find as a consequence of these considerations that if an anatic function $g(z)$ exists in $D$ with

$$
\lim _{n \rightarrow \infty} \oint_{C^{(n)}}|g(z)| d s=0, \quad g(\infty) \neq 0,
$$

theli no nonconstant bounded analytic function exists in $D$.

If $E$ consists of the interiors of a number of closed disjoint analytic curves $C_{1}, \cdots, C_{n}$, then we have

$$
\lim _{m \rightarrow \infty} K_{m}\left(z_{1}, z_{1}\right)=K\left(z_{1}, z_{1}\right)=\frac{\alpha}{2 \pi} .
$$


Thus it is to a certain degree natural to consider the rational function $K_{m}\left(z, z_{1}\right)$ to be the analogue of the $m$ th Tschebyscheff polynomial for the case of the problem of Painlevé. Here the mean modulus plays the role which the maximum modulus plays for the Tschebyscheff polynomials. The principal difficulty in this analogy is that the integral $L_{\psi}$ is not defined for a general domain $D$ complementary to a compact set $E$, so that one has to resort to the cumbersome process of exhaustion by interior domains.

An advantage of the result presented here is, however, that $K\left(z, z_{1}\right)$ is one of the most readily computed domain functions in the theory of conformal mapping for the case of smoothly bounded regions.

THEOREM 3. The conditions (127) and (134) are necessary and sufficient for the existence of a nonconstant bounded analytic function in the exterior $D$ of $a$ compact set $E$.

VI. The Pick-Nevanlinna theory of interpolation. We seek in this chapter to extend our results on Schwarz's lemma to obtain the analogue for multiplyconnected domains of the interpolation theory developed by Nevanlinna for simply-connected domains [20]. We succeed in doing this by combining the method we developed in Chapter III with a new improvement which Ahlfors has recently found of the method he employed in the paper [1]. It is to be noted that this combination of methods yields a most elegant and general formulation for the solution of problems of the type we treat in this paper.

We investigate the class $\Omega$ of analytic functions $F(z)$ in $D$ satisfying the conditions

$$
\begin{aligned}
& F\left(z_{i}\right)=t_{i}, \\
& i=1, \cdots, k^{\prime} \\
& |F(z)| \leqq 1, \\
& z \in D \text {. }
\end{aligned}
$$

We ask for the region of variation $\Delta$ of the value $F\left(z_{0}\right)$ at a $(k+1)$ st point $z_{0}$ of $D$ when $F$ ranges over the set $\Omega$.

$\Delta$ has a number of very simple properties which we immediately verify. Firstly, $\Delta$ is convex. For if $F_{1}\left(z_{0}\right)$ is in $\Delta$ and $F_{2}\left(z_{0}\right)$ is in $\Delta$, then clearly

$$
\mu_{1} F_{1}\left(z_{0}\right)+\mu_{2} F_{2}\left(z_{0}\right) \in \Delta, \quad \mu_{1}+\mu_{2}=1, \quad \mu_{1}, \mu_{2} \geqq 0,
$$

since $\left\{\mu_{1} F_{1}+\mu_{2} F_{2}\right\} \in \Omega$. Furthermore, $\Delta$ is convex with respect to all circles which have a point exterior to the unit circle. For if $t_{0}$ lies on the arc between $F_{1}\left(z_{0}\right)$ and $F_{2}\left(z_{0}\right)$ of the circle $\Gamma$ through the point $\tau_{0}$ exterior to the unit circle, we apply the preliminary transformation $T$ of the unit circle onto itself taking, in its analytic continuation, $\tau_{0}$ into the point at infinity. The function $F_{3}$ with $F_{3}\left(z_{0}\right)=t_{0}$ may now be defined by a relation

$$
F_{3}=T^{-1}\left\{\mu_{1} T\left(F_{1}\right)+\mu_{2} T\left(F_{2}\right)\right\},
$$

since $T$ carries $\Gamma$ into a straight line. $\Delta$ is closed by the theory of normal families, and we have shown that if $\Delta$ does not consist of a single point, and is 
not empty, then $\Delta$ has an interior point, which we again call $t_{0}$. For this is an evident consequence of the strong type of convexity we have established. We apply a map $T_{0}$ of the unit circle upon itself taking $t_{0}$ into the origin, and we maintain that $T_{0}(\Delta)$ is star-like with respect to the origin. This follows directly from the convexity.

We wish to investigate the extremal functions $F_{0}$ such that $F_{0}\left(z_{0}\right)$ lies on the boundary of $\Delta$. By applying the preliminary transformation $T_{0}$ and using the star-like property of $\Delta$ we see that it is sufficient to consider the problem of finding

$$
\max _{F \in \Omega_{0}}\left|F\left(z_{0}\right)\right|, \quad \arg F\left(z_{0}\right) \text { fixed, }
$$

and the associated extremal functions $F_{0}$. Here $\Omega_{0}$ refers to the transform of the class $\Omega$ under the transformation $T_{0}$. However, for the sake of simplifying the notation, and with no loss of generality, we shall take $\Omega_{0}$ now to be $\Omega$ itself.

Let $H(z ; \zeta)$ denote the conjugate harmonic function of the Green's function $G(z ; \zeta)$ of $D$. We suppose at first that there is a competing function $F(z)$ of the form

$$
F(z)=\exp \left(-\sum_{j=1}^{m}\left[G\left(z ; \zeta_{i}\right)+i H\left(z ; \zeta_{j}\right)\right]\right)
$$

for the problem (141). Then there is an extremal function $F_{0}$ of this type for the problem (141) posed in the subclass $\widetilde{\Omega}$ of $\Omega$ of functions $F$ of the type (142) with less than $Q$ zeros, for some large integer $Q$. Let (142) be this extremal function. Then, for points $\zeta_{j}^{*}$ near the $\zeta_{j}$ we have

$$
\sum_{j=1}^{m}\left[G\left(z_{0} ; \zeta_{j}^{*}\right)-G\left(z_{0} ; \zeta_{j}\right)\right] \geqq 0
$$

if

$$
\begin{array}{cc}
\sum_{j=1}^{m}\left[G\left(z_{i} ; \zeta_{j}^{*}\right)-G\left(z_{i} ; \zeta_{j}\right)\right]=0, & i=1, \cdots, k, \\
\sum_{j=1}^{m}\left[H\left(z_{i} ; \zeta_{j}^{*}\right)-H\left(z_{i} ; \zeta_{j}\right)-H\left(z_{0} ; \zeta_{j}^{*}\right)+H\left(z_{0} ; \zeta_{j}\right)\right]=0, i=1, \cdots, k, \\
\sum_{j=1}^{m}\left[\omega_{\nu}\left(\zeta_{j}^{*}\right)-\omega_{\nu}\left(\zeta_{j}\right)\right]=0, & \nu=1, \cdots, n-1 .
\end{array}
$$

We wish to rewrite the conditions (145) so that the $\zeta_{j}, \zeta_{j}^{*}$ appear as independent variables rather than as parameters. For this purpose we introduce the Neumann's function $N(x ; u, v)$ of $D . N(x ; u, v)$ is harmonic in $D$ except at $x=u$, where it has a positive logarithmic singularity, and at $x=v$, where 
it has a negative singularity, and

$$
\partial N / \partial n=0
$$

on $C$.

We denote by $\bar{N}(x ; u, v)$ the conjugate harmonic function of $N(x ; u, v) . N$ and $\bar{N}$ are determined up to constants which in no way affect our calculations. For the sake of definiteness we normalize so that $\oint_{c}(N+i \bar{N}) d s=0$. We draw a slit $\gamma_{i}$ from $z_{0}$ to each $z_{i}, i=1, \cdots, k$, and apply Green's theorem to the domains $D_{i}$ obtained from $D$ by deleting the slits $\gamma_{i}$. We have

$$
\begin{aligned}
H\left(z_{0} ; \zeta_{j}\right)-H\left(z_{i} ; \zeta_{j}\right) & =\frac{1}{2 \pi} \oint_{\gamma_{i}} \frac{\partial G\left(z ; \zeta_{j}\right)}{\partial n} \bar{N}\left(z ; z_{0}, z_{i}\right) d s \\
& =\frac{1}{2 \pi} \oint_{c} \frac{\partial G\left(z ; \zeta_{j}\right)}{\partial n} \bar{N}\left(z ; z_{0}, z_{i}\right) d s-\bar{N}\left(\zeta_{j} ; z_{0}, z_{i}\right),
\end{aligned}
$$

where the integral over $\gamma_{i}$ is to be extended over both sides of the slit. Now in consequence of (146) we have

$$
\frac{1}{2 \pi} \oint_{c}\left[\frac{\partial G\left(z ; \zeta_{j}^{*}\right)}{\partial n}-\frac{\partial G\left(z ; \zeta_{j}\right)}{\partial n}\right] \bar{N}\left(z ; z_{0}, z_{i}\right) d s=0,
$$

since $\bar{N}=$ const. on each $C_{\nu}$. Therefore we may replace the conditions (145) by the simpler conditions

$$
\sum_{j=1}^{m}\left[\bar{N}\left(\zeta_{i}^{*} ; z_{0}, z_{i}\right)-\bar{N}\left(\zeta_{j} ; z_{0}, z_{i}\right)\right]=0, \quad i=1, \cdots, k .
$$

Thus, as usual [7], variational formulas for $H$ lead to Neumann's function. We conclude by the results of Chapter II that there exist real parameters $\lambda_{i}, \mu_{i}$, and $\nu_{i}$, not all zero, such that

$$
h(z)=\sum_{i=1}^{k} \lambda_{i} G\left(z ; z_{i}\right)+\sum_{i=1}^{k} \mu_{i} \bar{N}\left(z ; z_{0}, z_{i}\right)+\sum_{i=1}^{n-1} \nu_{i} \omega_{i}(z)+\lambda_{0} G\left(z ; z_{0}\right)
$$

has critical points at $\zeta_{1}, \cdots, \zeta_{n}$. We define

$$
p^{\prime}(z)=\frac{\partial h}{\partial x}-i \frac{\partial h}{\partial y} \text {. }
$$

Since

$$
p^{\prime}(z) z^{\prime}(s)=\text { imaginary }
$$

on $C$,

we find from the argument principle that $p^{\prime}(z)$ has at most $(k+n-1)$ zeros in $D$.

Now to a zero of $F_{0}$ of order $\nu$ corresponds a critical point of $h$ of order at least $\nu$. For suppose $\zeta_{1}=\zeta_{2}=\cdots=\zeta_{\nu}=0$, say, and set 


$$
\begin{aligned}
& \zeta_{1}^{*}+\cdots+\zeta_{\nu}^{*}=\xi_{1}, \\
& \zeta_{1}^{*_{2}}+\cdots+\zeta_{\nu}^{* 2}=\xi_{2}, \\
& \cdots \cdots+\zeta_{\nu} \cdot \\
& \zeta_{1}^{*_{\nu}}+\cdots+\zeta_{\nu}^{*_{\nu}}=\xi_{\nu} .
\end{aligned}
$$

We consider the series expansion of

$$
\sum_{j=1}^{\infty} G\left(z_{0} ; \zeta_{i}^{*}\right)=\sum_{j=1}^{\nu} G\left(\zeta_{i}^{*} ; z_{0}\right)=\operatorname{Re}\left\{\nu \alpha_{0}+\sum_{i=1}^{\infty} \sum_{j=1}^{\infty} \alpha_{i} \zeta_{i}^{*_{i}}\right\},
$$

for example, and write

$$
\sum_{j=1}^{\nu} G\left(z_{0} ; \zeta_{i}^{*}\right)=\operatorname{Re}\left\{\nu \alpha_{0}+\alpha_{1} \xi_{1}+\cdots+\alpha_{\nu} \xi_{\nu}+\text { higher order terms }\right\} .
$$

Similar developments are to be found for the remaining terms in (143), (144), (146), and (150). We now see from (154), (155), (156) that we can introduce $\xi_{1}, \cdots, \xi_{\nu}$ as independent variables instead of $\zeta_{1}^{*}, \cdots, \zeta_{\nu}^{*}$. For if the $\beta_{i}$ are the symmetric functions of the $\zeta_{i}^{*}$ such that $\prod_{i=1}^{v}\left(t-\zeta_{i}^{*}\right)=t^{\nu}$ $-\beta_{1} t^{\nu-1}+\cdots+(-1)^{\nu} \beta_{\nu}$, then the $\xi_{i}$ are polynomials in the $\beta_{i}$ and the $\beta_{i}$ are polynomials in the $\xi_{i}$. Therefore any choice of the $\xi_{i}$ determines the $\beta_{i}$, which in turn determine the $\zeta_{i}^{*}$, and conversely. Furthermore, the functions $\sum_{i=1}^{\nu} \zeta_{i}^{* l}$ are of higher order than the first in $\xi_{1}, \cdots, \xi_{\nu}$ for $l>\nu$, so that (158) is justified. With power series representations of the form (158), the consideration of Chapter II show that the first $(\nu-1)$ derivatives of $p^{\prime}(z)$ vanish at $\zeta_{1}=\cdots=\zeta_{\nu}=0$. This method of Ahlfors proves, then, the statement we have made.

Thus $F_{0}$ has at most $(k+n-1)$ zeros, and these lie at the zeros of $p^{\prime}(z)$. We have tacitly assumed that no $t_{i}$ vanishes, and certainly a preliminary transformation of the unit circle can be made to bring this about. However, this is not necessary, since the problem becomes simpler if certain $t_{i}$ vanish. Indeed, the interpolation requirement at such a $z_{i}$ does not enter into the relations (143), (144), (146), and (150), for we have a logarithmic singularity at this point, and addition of finite terms does not destroy this singularity. In this case, therefore, $p^{\prime}(z)$ will have fewer critical points, while the bound on the number of zeros of $F_{0}$ will continue to be $(k+n-1)$.

We may now introduce a fictitious zero $\zeta_{0}$ of $F_{0}$ on the boundary $C$ of $D$. This is permissable if $Q$ is chosen greater than $(k+n-1)$. Since

$$
G\left(z ; \zeta_{0}\right) \equiv 0
$$

$$
z \in D, \zeta_{0} \in C,
$$

we can add a term in $\zeta_{0}$ to the variational equations (143), (144), (146), (150) with a varied position $\zeta_{0}^{*}$ of $\zeta_{0}$. We require only that $\zeta_{0}^{*}$ lie on the inner normal $n$ to $C$ at $\zeta_{0}$. This corresponds to conditions of the form (14) in Chapter II. We conclude that the signs of the $\lambda_{i}, \mu_{i}$, and $\nu_{i}$ can be chosen so 
that

$$
\partial h / \partial n \leqq 0
$$

on $C$.

We now set

$$
f_{0}(z)=p^{\prime}(z) / F_{0}(z) \text {. }
$$

$f_{0}$ is analytic in $D$ except for possible poles at $z_{0}, z_{1}, \cdots, z_{k}$. We have also

$$
\frac{f_{0}(z) F_{0}(z) z^{\prime}(s)}{i}=-\frac{\partial h}{\partial n} \geqq 0, \quad z \in C .
$$

Hence we have all the necessary tools for the application of the method of contour integration of Chapter III.

Let $F$ be a competing function of class $\Omega$ for the problem (141). We find

$$
\begin{aligned}
-\frac{\lambda_{0}}{F_{0}\left(z_{0}\right)}\left\{F_{0}\left(z_{0}\right)-F\left(z_{0}\right)\right\} & =\operatorname{Re}\left\{\frac{1}{2 \pi i} \oint_{C}\left(F_{0}-F\right) f_{0} d z\right\} \\
& =\frac{1}{2 \pi} \oint_{C}\left|f_{0}\right| d s-\operatorname{Re}\left\{\frac{1}{2 \pi i} \oint_{c} F f_{0} d z\right\} \geqq 0 .
\end{aligned}
$$

Now if $F \not \equiv F_{0}$, then $F \not \equiv F_{0}$ on $C$, and we have $\lambda_{0} \neq 0$. We obtain, indeed,

$$
-\lambda_{0} \operatorname{Re}\left\{\frac{F_{0}\left(z_{0}\right)-F\left(z_{0}\right)}{F_{0}\left(z_{0}\right)}\right\}>0 .
$$

Now $\arg F_{0}\left(z_{0}\right)=\arg F\left(z_{0}\right)$, and hence we have

$$
\left|F\left(z_{0}\right)\right|<\left|F_{0}\left(z_{0}\right)\right|, \quad \lambda_{0}<0
$$

or

$$
\left|F\left(z_{0}\right)\right|>\left|F_{0}\left(z_{0}\right)\right|, \quad \lambda_{0}>0 .
$$

In the case of the second alternative, the function $F_{0}(z)$ is the unique competing function of the type (142) for the problem (141), for if a second such function existed we could use it in the role of $F$ to arrive at a contradiction.

We have assumed so far that a competing function of the type (142) exists. We shall now justify this assumption and we shall also show that the alternative (166) never occurs. We proceed by induction upon the number $k$ of the interpolating conditions.

If $k=1$, we may assume without loss of generality that $t_{1}=0$. A competing function of the type (142) exists, and indeed we may use here the extremal function $F_{0}$ of Chapter III multiplied by a suitable factor of unit modulus. Also, $F_{0}^{2}$ multiplied by a suitable factor yields a competing function, and since we thus do not have uniqueness in the class of functions (142), the case (166) is impossible for $k=1$. A minor difficulty in this argument is found if $z_{0}$ is one of the zeros $z_{2}, \cdots, z_{n}$ of the extermal function of Chapter III. However, 
we may in this case take an extremal function for another point of normalization, and after a suitable linear transformation of the unit circle onto itself is made which leads to satisfaction of the interpolation condition, all goes through as before.

Hence for $k=1$ the extremal functions $F_{0}$ corresponding to boundary points of $\Delta$ have the form (142) with $m=n$, and they are unique. We suppose that for $k=k_{0}$ the same statement holds, except that we only have $m \leqq k_{0}$ $+n-1$. We prove the result for $k=k_{0}+1$.

If the first $k_{0}$ interpolation conditions are satisfied, then by the induction hypothesis the possible points $F\left(z_{k_{0+1}}\right)$ fill a region $\Delta$. If $t_{k_{0+1}}$ is not in $\Delta$, further interpolation is impossible and we are through. If $t_{k_{0+1}}$ lies on the boundary of $\Delta$, then there is only one possible interpolating function, and this has the form (142) with $m \leqq k_{0}+n-1$, by hypothesis. Again we are through. If $t_{k_{0}+1}$ lies interior to $\Delta$, the situation is slightly more complicated. We perform a transformation $T$ of the unit circle upon the upper half-plane. It is then possible to find interpolating functions of the form

$$
F=T^{-1}\left\{\mu_{1} T\left(F_{1}\right)+\mu_{2} T\left(F_{2}\right)\right\}, \quad \mu_{1}+\mu_{2}=1, \quad \mu_{1}, \mu_{2} \geqq 0,
$$

where $F_{1}$ and $F_{2}$ correspond to boundary points of $\Delta$. Here $\mu_{1}$ and $\mu_{2}$ are uniquely determined by $F_{1}, F_{2}$ and the condition $F\left(z_{k_{0}+1}\right)=t_{k_{0}+1}$. However, if we pick $\nu \geqq 4$ different functions $F_{1}, \cdots, F_{\nu}$ corresponding to boundary points of $\Delta$, then many different interpolating functions

$$
F=T^{-1}\left\{\sum_{j=1}^{\nu} \mu_{j} T\left(F_{j}\right)\right\}, \quad \sum_{j=1}^{\nu} \mu_{j}=1, \quad \mu_{1}, \cdots, \mu_{\nu} \geqq 0,
$$

may be set up. All the functions (168) will not be the same, since the $F_{j}$ are not identical on $C$ and the $T\left(F_{j}\right)$ have different poles there for a proper choice of $T$. We seek possible values $F\left(z_{0}\right)$ for $F$ 's of the form (142). There must be at least three of these, since the various functions (168) have the form (142). Indeed, if all $F\left(z_{0}\right)$ for $F$ 's of the type (168) coincide, then we may set up three problems of the type (141) after preliminary transformations $T_{1}, T_{2}$, and $T_{3}$ of the unit circle and thus obtain the three required different values, since the alternative (166) is excluded by the multiplicity of functions (168). On the other hand, if all $F\left(z_{0}\right)$ for $F$ 's of the type (168) do not coincide, then we may form combinations (167) of two of these $F$ 's and different choices of $T$ which lead to at least three different values $F\left(z_{0}\right)$. In both cases we get values not all on one line. By forming further combinations of our three interpolating functions in the upper half-plane, we obtain a triangle of values $F\left(z_{0}\right)$ with interpolating functions of the form (142). Thus clearly the alternative (166) cannot occur. Therefore, we find that the boundary of the region of values $F\left(z_{0}\right)$ corresponds to unique extremal functions of the form (142) with $m \leqq k_{0}+n$. Thus our induction proof is complete.

We remark that a generalized Jacobi inversion problem can be set up from 
the relation (162), the interpolation conditions, and the conditions that $F_{0}$ be single-valued. This generalized Jacobi problem is derived as in Chapter II, and, theoretically, it determines the zeros $\zeta_{1}, \cdots, \zeta_{m}$ of $F_{0}$. However, little is known about such inversion problems involving integrals of the third kind, and we do not go further into this question. However, it is worth noticing that many of the results of Chapter IV have their analogues in the present case. Thus we may express the functions $f_{0}$ and $F_{0}$ in terms of Green's and Neumann's functions and the harmonic measures by formulas like (76), (77), (83), (84), and (86).

THEOREM 4. Let $\Omega$ be the class of analytic functions $F(z)$ in $D$ satisfying (137) and (138), and let $\Delta$ be the set of values $F\left(z_{0}\right)$ for all $F \in \Omega$. Then $\Delta$ is $a$ closed convex set, and each function $F_{0}$ corresponding to a boundary point of $\Delta$ is unique and has the form (142) with $m \leqq k+n-1$. To an interior point of $\Delta$ correspond infinitely many interpolating functions $F \in \Omega$.

Furthermore, supposing for the sake of simplicity that no $t_{i}$ vanishes, the zeros $\zeta_{j}$ of $F_{0}$ lie at the critical points of a harmonic function (151) with nonpositive normal derivative. If we define $p^{\prime}(z)$ by the relation (152) and use (161) we have (162). $f_{0}(z)$ minimizes the integral

$$
\oint_{c}|f| d s
$$

among all functions $f(z)$, continuous in the closed neighborhood of $C$, which have the same poles at $z_{0}, z_{1}, \cdots, z_{k}$ as $f_{0}(z)$. If $\Delta$ has interior points, we have in all cases $\lambda_{0} \neq 0$.

We need now to prove that for all $F \in \Omega$ the integrals which we have taken over $C$ make sense. This follows from the fact that any $F \in \Omega$ has a nontangential limit almost everywhere on $C$ (see Nevanlinna [13]). Thus, using this limit to define $F$ almost everywhere on $C$, we may apply the Cauchy theorem to $F$ even on $C$. We have merely to use the Lebesgue theorem and a sequence of approximating contours to prove this statement. Thus our results apply in full rigor to the whole class $\Omega$.

The importance of Theorem 4 for the coefficient problem of bounded functions in multiply-connected domains is evident. Indeed, the reader will verify that interpolation conditions may be imposed upon the derivatives of $F$ as well as upon the values of the function itself. This leads to Schottky functions with higher order poles in place of the functions $G$ and $\bar{N}$ in the formulas (144), (150), and (151). Thus $p^{\prime}(z)$ will have higher order poles.

The coefficient problem has been treated in simply-connected domains by Carathéodory [6] and Schur [18]. We have succeeded in finding the extremal functions for this problem in multiply-connected domains and therefore we have a set of necessary and sufficient conditions upon the coefficients of the power series expansion of $F(z)$ at, say, $z_{0}$, in order that $|F(z)| \leqq 1, z \in D$. 
However, the geometry of the coefficient domain remains to be investigated, and we make no pretense at having a complete solution for multiply-connected domains.

VII. A further normalization. We have been able to solve a very general problem of interpolation for bounded functions, and it is clear that we could treat even more general cases with higher derivatives and poles entering into the picture. It is natural now to pose similar interpolation questions for the integrals (94) and (27) in view of our results in Chapters III and IV. The first case is quite simple to handle, for one merely sets up a linear combination of Szegö kernel functions

$$
\psi(z)=\sum_{j=1}^{m} \alpha_{j} K\left(z, z_{j}\right)
$$

satisfying the interpolation conditions $\psi\left(z_{j}\right)=t_{j}, j=1, \cdots, m$, and verifies by contour integration that this is the unique extremal function. For examples of a technique of this type, see Bergman [4] and Garabedian and Schiffer [7].

The second type of integral is far more difficult to handle. In order to show the difference between the integrals $l_{\sigma}$ and $L_{\psi}$ more clearly, we shall solve a specific minimum problem for $l_{g}$ in the class of analytic, rather than meromorphic, functions $g(z)$.

Let $R_{z_{1}}, z_{1} \in D$, be the class of analytic functions $g(z)$ in $D$, continuous in $D+C$, with $g\left(z_{1}\right)=1$. Let $\Lambda_{z_{1}}$ be the class of functions $E(z)$ in $D$ with one simple pole,

$$
E(z)=\frac{r_{E}}{z-z_{1}}+a_{0}+a_{1}\left(z-z_{1}\right)+\cdots, r_{E}>0,
$$

at $z=z_{1}$ and satisfying the relation

$$
\limsup _{z \rightarrow C}|E(z)| \leqq 1
$$

We pose the extremal problems

$$
\begin{aligned}
\lambda & =\min _{g \in R_{z_{1}}} l_{o}, \\
r & =\max _{E \in \Lambda_{z_{1}}} r_{E},
\end{aligned}
$$

and we try to find the associated extremal functions $g_{0}(z)$ and $E_{0}(z)$.

We proceed precisely as in Chapter VI, and therefore we give few details. First we find a function of the form

$$
E(z)=\exp \left(G\left(z ; z_{1}\right)+i H\left(z ; z_{1}\right)-\sum_{j=2}^{m}\left[G\left(z ; z_{j}\right)+i H\left(z ; z_{j}\right)\right]\right)
$$

in the class $\Lambda_{z_{1}}$. This is done by taking the reciprocal of the extremal func- 
tion $F_{0}(z)$ of Chapter III and multiplying it by suitable functions of the form (142), so that only the one pole at $z_{1}$ remains. Next we pose the problem (174) in the restricted class of functions (175) with $m \leqq Q$, where $Q$ is some sufficiently large fixed integer. This leads to an extremal function $E_{0}(z)$ of the type (175) with $m \leqq n-1$, whose zeros $z_{2}, \cdots, z_{m}$ lie at the critical points of a function

$$
\eta(z)=\mu_{0} G\left(z ; z_{1}\right)+\sum_{i=1}^{n-1} \mu_{i} \omega_{i}(z) .
$$

$\eta(z)$ is found by the method of Lagrange multipliers developed in Chapter II, and

$$
\partial \eta / \partial n \leqq 0
$$

on $C$.

Thus we may choose $\mu_{0}=-1$, and we set

$$
q^{\prime}(z)=\frac{\partial \eta}{\partial x}-i \frac{\partial \eta}{\partial y} .
$$

Finally, we set

$$
g_{0}(z)=r_{E_{0}} \frac{q^{\prime}(z)}{E_{0}(z)}
$$

Thus $g_{0}(z)$ satisfies a relation

$$
\frac{g_{0}(z) E_{0}(z) z^{\prime}(s)}{i} \geqq 0, \quad z \in C,
$$

and hence $g_{0}(z)$ is an extremal function for the problem (173), while $E_{0}$ is the extremal function for the problem (174). For

$$
\begin{aligned}
r_{E} & =\frac{1}{2 \pi i} \oint_{C} E(z) g(z) d z \\
& \leqq \frac{1}{2 \pi} \oint_{C}|g(z)| d s=\frac{l_{o}}{2 \pi},
\end{aligned}
$$

and equality holds if and only if $E \equiv E_{0}$ and

$$
\arg g(z) \equiv \arg g_{0}(z), \quad z \in C .
$$

We cannot show in general that the condition (181) determines $g(z)$ uniquely, and implies $g(z) \equiv g_{0}(z)$, since $E_{0}(z)$ can have less than $n-1$ zeros in $D$. However, if $E_{0}(z)$ does have $n-1$ zeros, then $\eta(z)$ and consequently $g_{0}(z)$ are uniquely determined. We have thus obtained a complete solution of the problem (173). A Jacobi inversion problem can be found by the methods of Chapter III for the determination of the zeros $z_{2}, \cdots, z_{m}$ of $E_{0}$. 
We introduce the functions

$$
\begin{aligned}
& P\left(z, z_{1}\right)=\frac{1}{r_{E_{0}}^{2}} \int_{z_{1}}^{z} g_{0}(\zeta) d \zeta, \\
& Q\left(z, z_{1}\right)=-\frac{1}{r_{E_{0}}^{2}} \int_{z_{1}}^{z} g_{0}(\zeta) E_{0}(\zeta)^{2} d \zeta
\end{aligned}
$$

and we have by (179)

$$
P^{\prime}\left(z, z_{1}\right) z^{\prime}=\overline{Q^{\prime}\left(z, z_{1}\right) z^{\prime}}
$$

Therefore $P$ and $Q$ can be represented in terms of Green's function and the harmonic measures. Also, we have

$$
E_{0}(z)^{2}=-Q^{\prime}\left(z, z_{1}\right) / P^{\prime}\left(z, z_{1}\right) .
$$

$P^{\prime}\left(z, z_{1}\right)$ has properties analogous to those of the Bergman kernel function. Thus differentials $d P$ and $d Q$ with the property $\overline{d P}=d Q$ on $C$ solve a very wide variety of extremal problems in conformal mapping.

\section{BIBLIOGRAPHY}

1. L. Ahlfors, Bounded analytic functions, Duke Math. J. vol. 14 (1947) pp. 1-11.

2. P. Alexandroff and H. Hopf, Topologie, I, Berlin, 1935.

3. S. Bergman, Partial differential equations, advanced topics, Brown University, Providence, 1941.

4. - Sur les fonctions orthogonales de plusieurs variables complexes avec les applications $d$ la theorie des fonctions analytiques, Paris, 1947.

5. S. Bergman and M. Schiffer, A representation of Green's and Neumann's functions in the theory of partial differential equations of second order, Duke Math. J. vol. 14 (1947) pp. 609638.

6. C. Caratheodory, Über den Variabilitätsbereich der Fourier'schen Konstanten von positiven harmonischen Funktionen, Rend. Circ. Mat. Palermo vol. 32 (1911) pp. 193-217.

7. P. Garabedian and M. Schiffer, Identities in the theory of conformal mapping, Trans. Amer. Math. Soc. vol. 65 (1949) pp. 187-238.

8. H. Grunsky, Neue Abschätzungen zur konformen Abbildung ein- und mehrfach zusammenhängenden Bereiche, Schriften des Mathematischen Seminars der Universität Berlin vol. 1 (1932) pp. 94-140.

9. - Eindeutige beschränkte Funktionen in mehrfach zusammenhängenden Gebieten, I-II, Jber. Deutschen Math. Verein. vol. 50 (1940) and vol. 52 (1942).

10. M. H. Heins, Extremal problems for functions analytic and single-valued in a doublyconnected region, Amer. J. Math. vol. 62 (1940) pp. 91-106.

11. - On a problem of Walsh concerning the Hadamard three circles theorem, Trans. Amer. Math. Soc. vol. 55 (1944) pp. 349-372. 1884.

12. C. Neumann, Vorlesungen über Riemann's Theorie der Abel'schen Integrale, Leipzig,

13. R. Nevanlinna, Eindeutige analytische Funktionen, Berlin, 1936.

14. R. M. Robinson, Analytic functions in circular rings, Duke Math. J. vol. 10 (1943) pp. 341-354.

15. M. Schiffer, The span of multiply connected domains, Duke Math. J. vol. 10 (1943) pp. 209-216. 
16. - The kernel function of an orthonormal system, Duke Math. J. vol. 13 (1946) pp. 529-540.

17. F. Schottky, Über die konforme Abbildung mehrfach zusammenhängender ebener Flächen, J. Reine Angew. Math. vol. 83 (1887) pp. 300-351.

18. J. Schur, Über Potenzreihen, die im Innern des Einheitskreises beschränkt sind, J. Reine Angew. Math. vol. 147 (1917) pp. 205-232 and vol. 148 (1918) pp. 122-145.

19. G. Szegö, Über orthogonale Polynome, die zu einer gegebenen Kurve der komplexen Ebene gehören, Math. Zeit. vol. 9 (1921) pp. 218-270.

20. J. L. Walsh, Interpolation and approximation by rational functions in the complex domain, Amer. Math. Soc. Colloquium Publications, vol. 20, New York. 1935.

HARVARD UNIVERSITY,

Cambridge, Mass. 